\title{
End-to-End Feature-aware Label Space Encoding for Multi- label Classification with Many Classes
}

\begin{tabular}{|r|l|}
\hline Journal: & IEEE Transactions on Neural Networks and Learning Systems \\
\hline Manuscript ID & TNNLS-2016-P-6192.R2 \\
\hline Manuscript Type: & Paper \\
\hline Date Submitted by the Author: & n/a \\
\hline Complete List of Authors: & $\begin{array}{l}\text { Lin, Zijia; Tsinghua University, Department of Computer Science and } \\
\text { Technology } \\
\text { Ding, Guiguang; Tsinghua University, School of Software } \\
\text { Han, Jungong; Northumbria University, Department of Computer Science } \\
\text { and Digital Technologies } \\
\text { Shao, Ling; Northumbria University, }\end{array}$ \\
\hline Keywords: & $\begin{array}{l}\text { Multi-label classification, Label space dimension reduction, End-to-end } \\
\text { feature-aware label space encoding, E2FE }\end{array}$ \\
\hline \multicolumn{2}{|c}{} \\
\hline
\end{tabular}


1

2

\section{Summary of Changes and Responses to Reviews}

We appreciate very much the handling editor and all reviewers for their time and efforts devoted to the peer-review of this paper. Their constructive comments have guided us to further improve it.

We have conducted a "minor revision" for the manuscript to address the editor's and reviewers' comments. And we believe that all the comments raised in the review report have been carefully accommodated. Below we will firstly list the changes we have made in this revision, and then respond to each comment in turn.

\section{Summary OF CHANGes}

The main changes made in this revision include:

1) In the "Introduction" of the revision, we have stated more clearly about the connection between our work and the community of neural networks and related learning systems, as well as our contributions to the community. Moreover, we have improved the language presentation of our manuscript with the help of a native speaker to make it more clear and easier to follow.

2) To make our manuscript more focused on the key points, we have moved the following content to a supplementary material: 1) proofs of Lemma 1 and Lemma 3, 2) overmuch details of the experiment for significance tests, and 3) overmuch details of the experiment for validating the orthonormality assumption in our proposed method. Particularly, for 2) and 3), we mainly present the corresponding experimental results and analysis in the manuscript. And for more details, one can refer to the supplementary material.

\section{RESPONSES TO REVIEWS}

1) Comment: The language presentation in this paper needs to be improved. The authors are encouraged to have a native speaker or use a professional editing service (see TNNLS website for more information, section "Professional Editing Services" under "Information for Authors") to help to improve the language presentation.

Response: Thanks for the suggestion. In the revision, we have invited our colleague, who is a native speaker and familiar with the topic, to help polish our manuscript and improve the language presentation.

2) Comment: The authors should clearly state the key contributions to the core of neural networks and related learning systems to show a strong connection to the NNLS community. The authors might want to take a look of the recently published papers in TNNLS on this topic as well.

Response: Thanks for the suggestion. In the "Introduction" of the revision, we have stated more clearly about the connection between our work and the NNLS community, as well as our contributions to the community. Please refer to the first two paragraphs of "Introduction" (Page 1) and the 5th paragraph of Page 2.

Specifically, multi-label classification, especially largescale multi-label classification, is an important topic for the machine learning community, with many research works like [1]-[7] dedicated to it for tackling challenges. Similar to the previous publications like [3], our work also focuses on tackling the challenge of large label sets in large-scale multi-label classification problems. To do so, we propose an effective method termed $\mathrm{E}^{2} \mathrm{FE}$ to perform label space dimension reduction (LSDR), which aims to yield acceptable classification performance with substantially lower costs. Extensive experiments show that $\mathrm{E}^{2} \mathrm{FE}$ gains performance improvements over other state-of-the-art LSDR methods. Moreover, since the predictive model used in $\mathrm{E}^{2} \mathrm{FE}$ is open for any effective one, including neural networks, $\mathrm{E}^{2} \mathrm{FE}$ can also be applied with existing predictive models or feature dimension reduction approaches in the community to better handle large-scale multi-label classification problems.

\section{REFERENCES}

[1] C. Ferng and H. Lin, "Multilabel classification using error-correcting codes of hard or soft bits," IEEE Transactions on Neural Networks and Learning Systems, vol. 24, no. 11, pp. 1888-1900, 2013.

[2] Y. Luo, D. Tao, C. Xu, C. Xu, H. Liu, and Y. Wen, "Multiview vectorvalued manifold regularization for multilabel image classification," IEEE Transactions on Neural Networks and Learning Systems, vol. 24, no. 5, pp. 709-722, 2013.

[3] F. Charte, A. Rivera, M. del Jesus, and F. Herrera, "Li-mlc: A label inference methodology for addressing high dimensionality in the label space for multilabel classification," IEEE Transactions on Neural Networks and Learning Systems, vol. 25, no. 10, pp. 1842-1854, 2014.

[4] W. Bi and J. Kwok, "Mandatory leaf node prediction in hierarchical multilabel classification," IEEE Transactions on Neural Networks and Learning Systems, vol. 25, no. 12, pp. 2275-2287, 2014.

[5] Q. Wu, Y. Ye, H. Zhang, T. Chow, and S. Ho, "Ml-tree: A tree-structurebased approach to multilabel learning," IEEE Transactions on Neural Networks and Learning Systems, vol. 26, no. 3, pp. 430-443, 2015.

[6] S. Chen, X. Yang, and Y. Tian, "Discriminative hierarchical k-means tree for large-scale image classification," IEEE Transactions on Neural Networks and Learning Systems, vol. 26, no. 9, pp. 2200-2205, 2015.

[7] X. Cao, C. Zhang, H. Fu, X. Guo, and Q. Tian, "Saliency-aware nonparametric foreground annotation based on weakly labeled data," IEEE Transactions on Neural Networks and Learning Systems, vol. 27, no. 6, pp. 1253-1265, 2016. 


\title{
End-to-End Feature-aware Label Space Encoding for Multi-label Classification with Many Classes
}

\author{
Zijia Lin, Student Member, IEEE, Guiguang Ding, Member, IEEE, \\ Jungong Han, Ling Shao, Senior Member, IEEE,
}

\begin{abstract}
To make the problem of multi-label classification with many classes more tractable, in recent years academia has seen efforts devoted to performing label space dimension reduction (LSDR). Specifically, LSDR encodes high-dimensional label vectors into low-dimensional code vectors lying in a latent space, so as to train predictive models at much lower costs. With respect to the prediction, it performs classification for any unseen instance by recovering a label vector from its predicted code vector via a decoding process. In this paper, we propose a novel method, namely End-to-End Feature-aware label space Encoding $\left(E^{2} F E\right)$, to perform LSDR. Instead of requiring an encoding function like most previous works, $\mathrm{E}^{2} \mathrm{FE}$ directly learns a code matrix formed by code vectors of the training instances in an endto-end manner. Another distinct property of $E^{2} F E$ is its feature awareness attributable to the fact that the code matrix is learnt by jointly maximizing the recoverability of the label space and the predictability of the latent space. Based on the learnt code matrix, $E^{2} F E$ further trains predictive models to map instance features into code vectors, and also learns a linear decoding matrix for efficiently recovering the label vector of any unseen instance from its predicted code vector. Theoretical analyses show that both the code matrix and the linear decoding matrix in $E^{2} F E$ can be efficiently learnt. Moreover, similar to previous works, $E^{2} \mathbf{F E}$ can be specified to learn an encoding function. And it can also be extended with kernel tricks to handle non-linear correlations between the feature space and the latent space. Comprehensive experiments conducted on diverse benchmark datasets with many classes show consistent performance gains of $E^{2} F E$ over the stateof-the-art methods.
\end{abstract}

Index Terms-End-to-end feature-aware label space encoding, Label space dimension reduction, Multi-label classification.

\section{INTRODUCTION}

A $\mathrm{S}$ a generalized version of multi-class classification [1], [2], where each instance is restricted to having only one class label, multi-label classification [3]-[20] allows an instance to be associated with several class labels to describe its semantic content or attributes more clearly. Multi-label classification methods are increasingly demanded by modern applications, like multi-label text classification [3], music emotion categorization [4], and semantic image annotation [18][20]. In addition, many researches on neural networks and

Zijia Lin is with the Department of Computer Science and Technology, Tsinghua University, Beijing 100084, China (e-mail: linzijia07etsinghua.org.cn).

Guiguang Ding is with School of Software, Tsinghua University, Beijing 100084, China (e-mail: dingggetsinghua.edu.cn). Guiguang Ding is the corresponding author.

Jungong Han is with the Department of Computer Science and Digital Technologies, Northumbria University, Newcastle, U.K. (e-mail: jungong.han@northumbria.ac.uk).

Ling Shao is with the School of Computing Sciences, University of East Anglia, Norwich NR4 7TJ, U.K. (e-mail: ling. shaodieee.org).

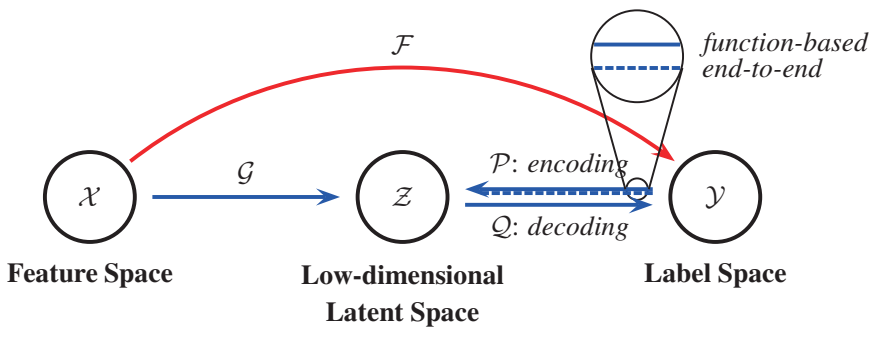

Fig. 1. Illustration of the principles behind traditional multi-label classification methods (red) and those with label space dimension reduction (blue).

other learning approaches are also dedicated to the field, like the tree-structure based method ML-TREE [17], the multiview vector-valued manifold regularization method $\mathrm{MV}^{3} \mathrm{MR}$ [18], and the label inference method LI-MLC [21], etc.

Recently, due to the emergence of web-based applications, multi-label classification problems tend to be large-scale, with new challenges of numerous instances and large label sets (i.e. high-dimensional label spaces) coming up. For instance, in the picture sharing community Flickr, there are billions of images and each can be annotated with textual labels selected from millions of candidates. In the community of neural networks and related learning systems, to handle the challenges, some works like [22]-[26] focus on feature dimension reduction or model simplification, while others like LI-MLC [21] focus on shrinking the label space. Here we follow the latter one.

As advocated by Kapoor et al. in [5], large label sets cause many existing effective multi-label classification methods [6][15] to be infeasible, since generally they need to learn a predictive model for each label independently or with interlabel correlations, and then combine them in a certain manner for prediction. Specifically, for a multi-label classification problem with many classes (i.e. a large label set, or a highdimensional label space), the number of needed predictive models would generally be large, thus making the training costs, if not unaffordable, extremely high. To tackle this issue, researchers have recently proposed to perform label space dimension reduction (LSDR) [5], [27]-[32], which aims to reduce the training costs while maintaining acceptable classification performance. Specifically, for LSDR, as illustrated in Fig. 1, the high-dimensional label vector of any training instance is encoded into a low-dimensional code vector in a latent space. Afterwards, predictive models are trained to map instance features into low-dimensional code vectors, whose quantity is much smaller and thus can significantly reduce the 
training costs. As for performing prediction for any unseen instance, a low-dimensional code vector is firstly obtained with the learnt predictive models from its features, and then decoded for recovering its label vector. Generally speaking, if the learnt predictive models and the decoding process are effective and efficient enough, LSDR usually yields acceptable classification performance with much lower costs, making the multi-label classification problem with many classes more tractable.

Prior methods dedicated to LSDR mostly require an encoding function (function-based), e.g. a linear one, to map label vectors of training instances into code vectors lying in the latent space. However, due to the following observations, we argue that learning the code vectors of training instances in an end-to-end manner, i.e. directly learning them without any encoding functions, can be feasible and even preferable.

- From Fig. 1 it can be seen that, to perform prediction, the encoding process is totally redundant, and thus any encoding function is useless during prediction. Moreover, even for training, it is the encoding result (i.e. code vectors of training instances) that will affect the learning of predictive models, no matter whether an encoding function is required or not.

- Defining an encoding function may limit the searching space of the to-be-learnt code vectors of training instances. For example, given the tagging matrix $\mathbf{Y}$ of training instances, using a linear encoding function $\mathbf{P}$ can limit the to-be-learnt code vectors in the space $\mathbf{Y P}$, thereby preventing them from being searched in the whole real space that could potentially minimize the loss of classification performance.

- In some cases, code vectors of training instances are required to have specific properties, like the orthonormality between code dimensions in this paper. Although those property requirements can somehow be transferred to the encoding function, it will inevitably make the objective function much more complex for optimization.

In fact, compared to a function-based encoding, an endto-end encoding requires no encoding function, and thus can search the whole real space for the optimal to-be-learnt code vectors. Moreover, for an end-to-end encoding, it would be direct to add property requirements for the to-be-learnt code vectors, making the objective function less complex for optimization. To the best of our knowledge, MLC-BMaD [31] is the only previous research that pioneered end-to-end label space encoding via boolean matrix decomposition. However, as will be shown later, its training is not efficient enough and as a result it may not fully accomplish the goal of LSDR. Moreover, MLC-BMaD learns the code vectors of training instances in a feature-unaware manner, meaning that the correlations between the latent space and the feature space are not considered. That, as advocated by Chen and Lin [30], can probably make the learnt latent space less predictable and thus degrade the final classification performance. Therefore, further researches on end-to-end label space encoding are highly expected.

In this paper, we propose a novel method termed $\mathrm{E}^{2} \mathrm{FE}$ to perform LSDR via End-to-End Feature-aware label space Encoding. Specifically, $E^{2} F E$ directly learns a code matrix formed by code vectors of training instances via jointly maximizing the recoverability of the label space and the predictability of the latent space, with the latter considering the correlations between the latent space and the feature space. And thus $\mathrm{E}^{2} \mathrm{FE}$ is feature-aware. Based on the learnt code matrix, predictive models are trained as other LSDR methods, to predict code vectors from instance features. Meanwhile, $\mathrm{E}^{2} \mathrm{FE}$ further learns a linear decoding matrix that can recover the predicted label vector of any unseen instance from its code vector generated by the trained predictive models.

Since the predictive models in the proposed $\mathrm{E}^{2} \mathrm{FE}$ are open for any effective ones, including neural networks, $\mathrm{E}^{2} \mathrm{FE}$ can actually be applied with existing predictive models or feature dimension reduction approaches in the community to better tackle the large-scale multi-label classification problem. Particularly for LSDR, below are three highlighted properties of $\mathrm{E}^{2} \mathrm{FE}$, which are in line with our contributions.

- We propose an effective LSDR method termed $\mathrm{E}^{2} \mathrm{FE}$ for tackling (large-scale) multi-label classification problems with many classes. To the best of our knowledge, it is the first to make LSDR both end-to-end and feature-aware.

- We jointly maximize the recoverability of the label space and the predictability of the latent space for performing LSDR in $\mathrm{E}^{2} \mathrm{FE}$. The objective function w.r.t the to-belearnt code matrix can be transformed to an eigenvalue problem, and is sufficiently flexible in the sense that different optimization strategies can be used depending on the applications for efficient optimization.

- We show that $\mathrm{E}^{2} \mathrm{FE}$ is a generic approach that covers previous LSDR researches, and it can also be specified to learn an encoding function. Moreover, it can be extended with kernel tricks to handle non-linear correlations between the feature space and the latent space.

This paper is based on our previous work presented in [33], which was termed FaIE, but it substantially extends that work by enhancing the proposed method to be more efficient and effective. Below are the summarized extensions.

- We propose a more efficient optimization method for the proposed method to learn the code matrix in cases where $n \gg d_{x}+d_{y}$, with $n, d_{y}, d_{x}$ respectively denoting the number of training instances, the dimensionality of the label space and that of the feature space. This is helpful for practical applications, as such cases are quite common. Specifically, the newly proposed optimization method transforms the size of the eigenvalue problem w.r.t the objective function of $\mathrm{E}^{2} \mathrm{FE}$ from $\mathbb{R}^{n \times n}$ to $\mathbb{R}^{\left(d_{x}+d_{y}\right) \times\left(d_{x}+d_{y}\right)}$, which can be solved more efficiently and can substantially reduce space costs.

- We further propose $\pi \mathrm{E}^{2} \mathrm{FE}, \pi \mathrm{LinearE}^{2} \mathrm{FE}$ and kernel$\pi \mathrm{E}^{2} \mathrm{FE}$, to consider the priori knowledge provided by the eigenvalue problem w.r.t the to-be-learnt code matrix for learning an enhanced decoding matrix. Experiments comparing $\pi \mathrm{E}^{2} \mathrm{FE}, \pi$ LinearE ${ }^{2} \mathrm{FE}$, kernel- $\pi \mathrm{E}^{2} \mathrm{FE}$ with their corresponding counterparts, i.e. $\mathrm{E}^{2} \mathrm{FE}$, LinearE ${ }^{2} \mathrm{FE}$ and kernel- $\mathrm{E}^{2} \mathrm{FE}$, show that enhancing the decoding matrix 
with such priori knowledge can help to gain significant performance improvements (on average $48.1 \%$ for labelbased macroFl and $33.9 \%$ for example-based Accuracy).

- In this paper, we provide a thorough discussion and experimental validation for that the orthonormality assumption for columns of the to-be-learnt code matrix in $\mathrm{E}^{2} \mathrm{FE}$ is reasonable. We also make error analyses for the proposed $\mathrm{E}^{2} \mathrm{FE}$, and derive its error bound. Additionally, more theoretical analyses, like those regarding time complexity and parameter settings, are also presented here.

- To better validate the effectiveness of $E^{2} \mathrm{FE}$, we utilize more widely-used benchmark datasets for experiments. We also conduct the experiments on the full datasets instead of the sampled ones in [33], so as to demonstrate the applicability of $\mathrm{E}^{2} \mathrm{FE}$ for handling larger datasets. More experimental results are also reported, like the significance tests for the improvements gained by $\mathrm{E}^{2} \mathrm{FE}$ over compared baselines, and the comparison of computational costs between the newly proposed optimization method here and that presented in [33].

The remainder of this paper is organized as follows. Section II gives an overview of related works. Section III elaborates on the proposed $E^{2} F E$. Section IV shows the proposed optimization methods and its corresponding theoretical analyses. Section V describes details about enhancing the linear decoding matrix with priori knowledge. Then Section VI presents the extensions of $\mathrm{E}^{2} \mathrm{FE}$, and analyses its relations to previous works. Experimental settings, results and analyses are given in Section VII. Finally we present discussions regarding $\mathrm{E}^{2} \mathrm{FE}$ in Section VIII and conclude the paper in Section IX.

\section{RELATED WORK}

With the explosion of label spaces in real-world applications, many remarkable effective multi-label classification methods tend to be infeasible due to the high training costs. To tackle such multi-label classification problems with many classes, a lot of effective methods were proposed, like constructing a hierarchy of multi-label classifiers [34], refining the output of heuristic efficient classifiers [35], performing label selection to recover the vocabulary with only a subset [36], or using label inference method based on the use of association rules to discover label dependencies [21], etc. Recently, LSDR was also proposed and is attracting more and more attention.

To the best of our knowledge, Hsu et al. [27] are the first to propose LSDR. Specifically, Hsu et al. exploited the sparsity of the label space, and proposed to linearly encode it to a lowdimensional latent space as compressed sensing (CS) and then train linear regression models w.r.t the derived codes. As for performing classification for an unseen instance, a code vector is firstly obtained with the learnt regression models from its features and then decoded with standard recovery algorithms like CoSaMP [37] to derive the predicted label vector. Kapoor et al. [5] further considered both label space compression and predictive model learning in a single probabilistic model, and derived a Bayesian framework termed BML-CS for multi-label classification via jointly optimizing over both.

Apart from compressed sensing based methods, Tai and Lin [28] proposed to perform principle label space transformation
TABLE I CATEgORIZATION OF EXISTING LSDR METHODS AND $\mathrm{E}^{2} \mathrm{FE}$

\begin{tabular}{c|c|c} 
& feature-unaware & feature-aware \\
\hline \multirow{2}{*}{ function-based } & CS [27], PLST [28], & BML-CS [5], \\
& CL [29], ML-CSSP [32] & CPLST [30] \\
\hline end-to-end & MLC-BMaD [31] & E $^{2}$ FE \\
\hline
\end{tabular}

(PLST) for seeking important correlations between labels, which is essentially PCA [38] for the label space. Chen and Lin [30] further enhanced it by proposing feature-aware conditional principal label space transformation (CPLST), which actually integrates orthogonally constrained canonical correlation analysis into the framework of PLST for considering the predictability of the latent space. Both PLST and CPLST performed LSDR via linear encoding and linear decoding. Zhou et al. [29] proposed another method termed "Compressed Labelling (CL)", which takes the signs of the linear Gaussian random projection results on the original label vectors as the derived code vectors and utilizes a series of Kullback-Leibler divergence based hypothesis tests for decoding. Alternatively, Wicker et al. [31] proposed MLC-BMaD for LSDR via boolean matrix decomposition on the binary tagging matrix, factorizing it as the product of a binary code matrix and a binary linear decoding matrix. Bi and Kwok [32] presented an efficient randomized sampling procedure termed ML-CSSP for selecting a column subset of the tagging matrix that can well span it, which is a special case of linear encoding.

Actually, the majority of existing methods perform LSDR in a function-based manner and require an encoding function. Such approaches, as analysed in section I, carry several drawbacks. To avoid those, performing LSDR in an endto-end manner with no need for any encoding function is highly desired. MLC-BMaD seems to be the only existing LSDR method that supports end-to-end label space encoding via boolean matrix factorization. However, MLC-BMaD is feature-unaware, and thus the learnt latent space could be less predictable, which can result in performance deterioration. Therefore, in this paper we propose $\mathrm{E}^{2} \mathrm{FE}$, which performs LSDR in an end-to-end manner and is also feature-aware.

To sum up, Table I categorizes the remarkable existing LSDR methods and the proposed $\mathrm{E}^{2} \mathrm{FE}$ into different combinations of $\{$ function-based, end-to-end $\}$ and ffeature-unaware, feature-aware $\}$, which well highlights the distinctness of $\mathrm{E}^{2} \mathrm{FE}$.

\section{PROPOSED APPROACH}

\section{A. Preliminaries}

Generally in the case of multi-label classification, the features of an instance are represented as a $d_{x}$-dimensional feature vector $\mathbf{x}$ in the feature space $\mathcal{X}$, i.e. $\mathbf{x} \in \mathcal{X} \subset \mathbb{R}^{d_{x}}$, and its labels are represented as a $d_{y}$-dimensional binary label vector $\mathbf{y}$ in the label space $\mathcal{Y}$, i.e. $\mathbf{y} \in \mathcal{Y} \subset\{0,1\}^{d_{y}}$. Here the $i$ th entry of the label vector $\mathbf{y}$ is set as 1 if the instance is associated with the $i$ th label and 0 otherwise. Suppose that we are given $n$ labelled instances for training, denoted as $\left\{\left(\mathbf{x}^{(i)}, \mathbf{y}^{(i)}\right)\right\}_{i=1}^{n}$, with $\mathbf{x}^{(i)}$ and $\mathbf{y}^{(i)}$ being the feature vector and the label vector of the $i$ th training instance. 
Multi-label classification will utilize them to learn the mapping $\mathcal{F}: \mathcal{X} \rightarrow \mathcal{Y}$ from the feature space $\mathcal{X}$ to the label space $\mathcal{Y}$, as illustrated in Fig. 1, and then utilize $\mathcal{F}$ for predicting the label vector of any unseen instance based on its feature vector.

As mentioned before, to derive the mapping $\mathcal{F}$, many existing effective multi-label classification methods will learn a predictive model for each label independently or with interlabel correlations, and then combine them in a certain manner for prediction. In that case, the number of the to-be-learnt predictive models will be at least $d_{y}$, and even much larger for methods using label powerset [39]. Then for a multilabel classification problem with many classes, $d_{y}$ will become quite large and the training costs of the to-be-learnt predictive models will be extremely high and even unaffordable. To tackle such a challenge, LSDR was recently proposed and is attracting more and more attention. With LSDR, the training process to learn $\mathcal{F}$ is transformed into a two-step learning process. That is, firstly the label vectors of training instances are encoded into low-dimensional code vectors in a latent space $\mathcal{Z} \subset \mathbb{R}^{d_{z}}$ with an encoding process $\mathcal{P}: \mathcal{Y} \mapsto \mathcal{Z}$, and then a mapping $\mathcal{G}: \mathcal{X} \mapsto \mathcal{Z}$ w.r.t the code vectors is learnt. Here $d_{z}$ is the dimensionality of the latent space $\mathcal{Z}$, and generally $d_{z} \ll d_{y}$. Moreover, as illustrated in Fig. $1, \mathcal{P}$ can be performed in a function-based manner (e.g. linear encoding function) or an end-to-end manner (e.g. matrix decomposition). Similar to learning $\mathcal{F}$, learning $\mathcal{G}$ can be based on training $d_{z}$ predictive models, one for a dimension of $\mathcal{Z}$. As for predicting the labels of any unseen instance, a $d_{z}$ dimensional code vector in $\mathcal{Z}$ will firstly be derived using the learnt $\mathcal{G}$ with its feature vector, and then a $d_{y}$-dimensional predicted label vector will be recovered through a decoding process $\mathcal{Q}: \mathcal{Z} \mapsto \mathcal{Y}$. For LSDR methods, with $d_{z} \ll d_{y}$, the number of the to-be-learnt predictive models is generally much smaller and thus the training costs are substantially lowered, making the multi-label classification problem with many classes more tractable. Meanwhile, if the mapping $\mathcal{G}$ and the decoding process $\mathcal{Q}$ are effective enough, the classification performance using LSDR is expected to be acceptable.

It should be noticed that for LSDR, the latent space $\mathcal{Z}$ is supposed to be derived from the label space $\mathcal{Y}$ rather than the feature space $\mathcal{X}$, even though $\mathcal{X}$ can sometimes be considered for increasing the predictability of $\mathcal{Z}$. And thus the dimensionality of the latent space $\left(\right.$ i.e. $d_{z}$ ) can either be higher or lower than that of the feature space (i.e. $d_{x}$ ), but will always be lower than that of the label space (i.e. $d_{y}$ ). Moreover, for LSDR methods, the mapping $\mathcal{G}$ from $\mathcal{X}$ to $\mathcal{Z}$ is open for any effective mapping algorithm after $\mathcal{Z}$ is derived. Meanwhile, the decoding process $\mathcal{Q}$ generally needs to be specified before deriving $\mathcal{Z}$, which, from the perspective of efficiency in prediction, is preferred to be linear, like those in PLST, CPLST, MLC-BMad and ML-CSSP.

\section{B. End-to-End Feature-aware Label Space Encoding}

Before elaborating on the proposed $\mathrm{E}^{2} \mathrm{FE}$, to make it more clear, Table II summarizes the important symbols in this paper.

As mentioned previously, $E^{2} \mathrm{FE}$ performs LSDR in an endto-end manner and directly learns a code matrix $\mathbf{Z} \in \mathbb{R}^{n \times d_{z}}$
TABLE II IMPORTANT SYMBOLS IN THE PROPOSED E ${ }^{2} \mathrm{FE}$

\begin{tabular}{l|l}
\hline$n$ & the number of training instances \\
\hline$d_{x}$ & the dimensionality of the feature space $\mathcal{X}$ \\
\hline$d_{y}$ & the dimensionality of the label space $\mathcal{Y}$ \\
\hline$d_{z}$ & the dimensionality of the latent space $\mathcal{Z}, d_{z} \ll d_{y}$ \\
\hline $\mathbf{X}$ & the feature matrix of training instances, $\mathbf{X} \in \mathbb{R}^{n \times d_{x}}$ \\
\hline $\mathbf{Y}$ & the tagging matrix of training instances, $\mathbf{Y} \in\{0,1\}^{n \times d_{y}}$ \\
\hline $\mathbf{Z}$ & the code matrix of training instances, $\mathbf{Z} \in \mathbb{R}^{n \times d_{z}}$ \\
\hline $\mathbf{Q}$ & the linear decoding matrix, $\mathbf{Q} \in \mathbb{R}^{d_{z} \times d_{y}}$ \\
\hline $\mathbf{H}$ & notation for $\mathbf{X}\left(\mathbf{X}^{T} \mathbf{X}\right)^{-1} \mathbf{X}^{T}, \mathbf{H} \in \mathbb{R}^{n \times n}$ \\
\hline $\mathbf{M} \cdot, i$ & the $i$ th column of a matrix $\mathbf{M}$ \\
\hline
\end{tabular}

formed by code vectors of training instances. Generally, the classification performance of LSDR methods depends on both the predictive mapping $\mathcal{G}$ and the decoding process $\mathcal{Q}$. Therefore, it is crucial for code vectors to be predictable, having a strong correlation with instance features, as revealed in [40]. Meanwhile, the label vectors should also be highly recoverable via decoding the corresponding code vectors. Therefore, to learn $\mathrm{Z}, \mathrm{E}^{2} \mathrm{FE}$ jointly maximizes the recoverability of the label space and the predictability of the latent space. The former is denoted as $\Psi_{1}(\mathbf{Y}, \mathbf{Z})$ and the latter as $\Psi_{2}(\mathbf{X}, \mathbf{Z})$, where $\mathbf{Y} \in\{0,1\}^{n \times d_{y}}$ is the tagging matrix of training instances formed by their label vectors row by row and $\mathbf{X} \in \mathbb{R}^{n \times d_{x}}$ is the feature matrix formed by their feature vectors in the same way. Then the objective function w.r.t $\mathbf{Z}$ is as follows.

$$
\Psi=\max _{\mathbf{Z}} \Psi_{1}(\mathbf{Y}, \mathbf{Z})+\alpha \Psi_{2}(\mathbf{X}, \mathbf{Z})
$$

where $\alpha \geq 0$ is a parameter for balancing recoverability and predictability. When $\alpha=0, \mathbf{Z}$ will be derived via merely maximizing recoverability, implying that $\mathbf{Z}$ is just dependent on $\mathbf{Y}$. On the contrary, when $\alpha>0$, correlations between instance features and code vectors will be further considered for making $\mathbf{Z}$ feature-aware and more predictable.

1) Recoverability of Label Space: To improve the recoverability of the label space, the difference between the tagging matrix $\mathbf{Y}$ and the recovered one, which is based on the tobe-learnt code matrix $\mathbf{Z}$, is expected to be minimized. Here we denote the difference as $\mathcal{L}$. As mentioned previously, for efficient decoding, the proposed $\mathrm{E}^{2} \mathrm{FE}$ learns a linear decoding matrix $\mathbf{Q} \in \mathbb{R}^{d_{z} \times d_{y}}$ to recover label vectors from code vectors, following PLST, CPLST, MLC-BMad and ML-CSSP. Then $\mathcal{L}$ is formulated as follows.

$$
\mathcal{L}=\min \|\mathbf{Y}-\mathbf{Z Q}\|_{\text {fro }}^{2}
$$

where $\|\cdot\|_{\text {fro }}$ is the Frobenius norm of a matrix. Given $\mathbf{Z}$, the optimal $\mathbf{Q}$ to minimize $\mathcal{L}$ can be derived as the following closed-form expression by solving $\frac{\partial \mathcal{L}}{\partial \mathbf{Q}}=\mathbf{0}$.

$$
\mathbf{Q}=\left(\mathbf{Z}^{T} \mathbf{Z}\right)^{-1} \mathbf{Z}^{T} \mathbf{Y}
$$

To mitigate redundant information in the latent space and then encode the label space more compactly, we assume that the dimensions of the latent space are uncorrelated and thus the columns of $\mathbf{Z}$ are orthonormal, as shown in formula (4).

$$
\mathbf{Z}^{T} \mathbf{Z}=\mathbf{I}
$$


where $\mathbf{I} \in \mathbb{R}^{d_{z} \times d_{z}}$ is an identity matrix. Actually, as analysed later, although such an orthonormality assumption may seem to be strong, it is still reasonable and important for $\mathrm{E}^{2} \mathrm{FE}$. With formula (4), the optimal $\mathbf{Q}$ can be simplified as $\mathbf{Q}=\mathbf{Z}^{T} \mathbf{Y}$, and then formula (2) can be reformulated as follows.

$$
\mathcal{L}=\operatorname{Tr}\left[\mathbf{Y}^{T} \mathbf{Y}-\mathbf{Y}^{T} \mathbf{Z} \mathbf{Z}^{T} \mathbf{Y}\right]
$$

where $\operatorname{Tr}[\cdot]$ refers to the trace of a matrix. With $\operatorname{Tr}\left[\mathbf{Y}^{T} \mathbf{Y}\right]$ being a constant, minimizing $\mathcal{L}$ is identical to maximizing $\operatorname{Tr}\left[\mathbf{Y}^{T} \mathbf{Z} \mathbf{Z}^{T} \mathbf{Y}\right]$, which can be seen as an expression of the recoverability of the label space, i.e. $\Psi_{1}(\mathbf{Y}, \mathbf{Z})$. We can thus derive the following formula.

$$
\begin{aligned}
& \Psi_{1}(\mathbf{Y}, \mathbf{Z})=\operatorname{Tr}\left[\mathbf{Y}^{T} \mathbf{Z} \mathbf{Z}^{T} \mathbf{Y}\right]=\operatorname{Tr}\left[\mathbf{Z}^{T} \mathbf{Y} \mathbf{Y}^{T} \mathbf{Z}\right] \\
& \text { s.t. } \quad \mathbf{Z}^{T} \mathbf{Z}=\mathbf{I}
\end{aligned}
$$

2) Predictability of Latent Space: As advocated in [40], to improve the predictability of the latent space, the code matrix $\mathbf{Z}$ is supposed to be strongly correlated with the instance features. Here we firstly consider linear correlations, and will later handle non-linear ones with kernel tricks. Considering a linear projection $\mathbf{w}$ for the feature space and a dimension $\mathbf{z}$ of the latent space, i.e. a column of $\mathbf{Z}$, the correlation between features and $\mathbf{z}$, denoted as $r(\mathbf{X}, \mathbf{z})$, can be defined as follows.

$$
r(\mathbf{X}, \mathbf{z})=\frac{(\mathbf{X w})^{T} \mathbf{z}}{\sqrt{(\mathbf{X w})^{T}(\mathbf{X w})} \sqrt{\mathbf{z}^{T} \mathbf{z}}}
$$

Due to the orthonormality assumption for $\mathbf{Z}$, i.e. formula (4), $\mathbf{z}^{T} \mathbf{z}=1$ will hold for any column of $\mathbf{Z}$. Moreover, linearly rescaling $\mathbf{w}$ by a non-zero multiplier will not change $r(\mathbf{X}, \mathbf{z})$. Then maximizing $r(\mathbf{X}, \mathbf{z})$ equals the following formula.

$$
\max (\mathbf{X w})^{T} \mathbf{z} \quad \text { s.t. } \quad(\mathbf{X w})^{T} \mathbf{X} \mathbf{w}=1
$$

Given a dimension $\mathbf{z}$ of the latent space, the maximal $r(\mathbf{X}, \mathbf{z})$ reflects its potential maximal correlation with the feature space, and thus the maximal $r(\mathbf{X}, \mathbf{z})$ can be seen as an expression of the predictability of $\mathbf{z}$. Specifically, with $\mathbf{z}$ fixed, the optimal $\mathbf{w}$ for formula (8), denoted as $\mathbf{w}^{*}$, can be derived as follows with the method of Lagrange multipliers.

$$
\mathbf{w}^{*}=\frac{\left(\mathbf{X}^{T} \mathbf{X}\right)^{-1} \mathbf{X}^{T} \mathbf{z}}{\sqrt{\mathbf{z}^{T} \mathbf{X}\left(\mathbf{X}^{T} \mathbf{X}\right)^{-1} \mathbf{X}^{T} \mathbf{z}}}
$$

Note that following CPLST, here we assume $\mathbf{A}=\mathbf{X}^{T} \mathbf{X}$ to be invertible. Actually, this assumption usually holds when $n>d_{x}$, but it will fail in cases with $n<d_{x}$, as $\mathbf{A}$ will not be full-rank then. To handle the latter cases, we propose to ensure $\mathbf{A}$ to be invertible via: 1$)$ performing dimensionality reduction for the feature space via PCA or alternative methods to make $d_{x}$ small enough for obtaining a full-rank $\mathbf{X}^{T} \mathbf{X}$, or 2) adding a tiny value to the entries on the diagonal of $\mathbf{X}^{T} \mathbf{X}$, i.e. $\mathbf{A}=\mathbf{X}^{T} \mathbf{X}+\varepsilon \mathbf{I}_{1}$ with $\mathbf{I}_{1} \in \mathbb{R}^{d_{x} \times d_{x}}$ being an identity matrix and $\varepsilon$ being a tiny value, e.g. $10^{-6}$.

By substituting $\mathbf{w}^{*}$ into formula (7), the predictability of $\mathbf{z}$, denoted as $\psi_{2}(\mathbf{X}, \mathbf{z})$, can be derived as follows.

$$
\psi_{2}(\mathbf{X}, \mathbf{z})=\frac{\left(\mathbf{X w}^{*}\right)^{T} \mathbf{z}}{\sqrt{\left(\mathbf{X w}^{*}\right)^{T}\left(\mathbf{X w}^{*}\right)} \sqrt{\mathbf{z}^{T} \mathbf{z}}}=\left(\mathbf{X} \mathbf{w}^{*}\right)^{T} \mathbf{z}=\sqrt{\mathbf{z}^{T} \mathbf{H z}}
$$

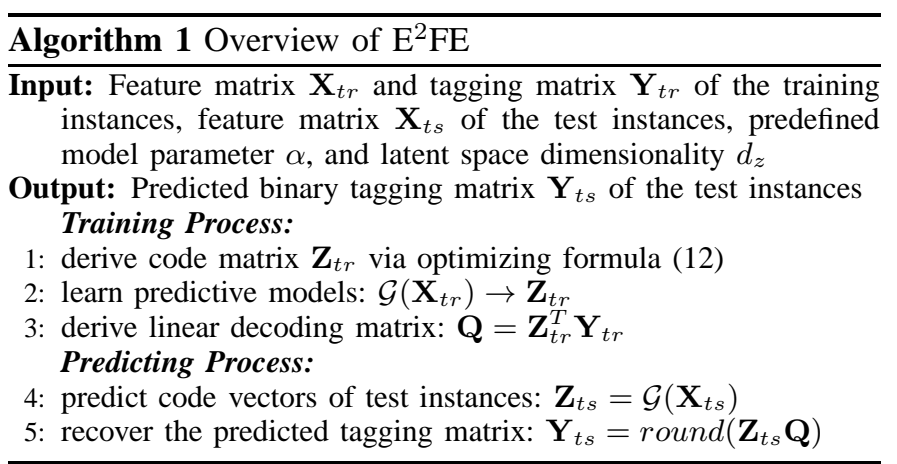

where $\mathbf{H}=\mathbf{X}\left(\mathbf{X}^{T} \mathbf{X}\right)^{-1} \mathbf{X}^{T} \in \mathbb{R}^{n \times n}$. To improve the predictability of the latent space, each column $\mathbf{z}$ of the code matrix $\mathbf{Z}$ is supposed to maximize $\psi_{2}(\mathbf{X}, \mathbf{z})$. As maximizing $\psi_{2}(\mathbf{X}, \mathbf{z})$ can be guaranteed by maximizing $\mathbf{z}^{T} \mathbf{H z}$, the overall predictability of $\mathbf{Z}$ can be formulated as follows.

$$
\begin{aligned}
& \Psi_{2}(\mathbf{X}, \mathbf{Z})=\sum_{i=1}^{d_{z}} \mathbf{Z}_{\cdot, i}^{T} \mathbf{H Z}{ }_{, i}=\operatorname{Tr}\left[\mathbf{Z}^{T} \mathbf{H Z}\right] \\
& \text { s.t. } \quad \mathbf{Z}^{T} \mathbf{Z}=\mathbf{I}
\end{aligned}
$$

where $\mathbf{Z}_{, i}\left(i \in\left\{1,2, \ldots, d_{z}\right\}\right)$ denotes the $i$ th column of $\mathbf{Z}$.

3) Detailed Objective Function: With $\Psi_{1}(\mathbf{Z}, \mathbf{Y})$ and $\Psi_{2}(\mathbf{X}, \mathbf{Z})$ derived, the objective function w.r.t the to-be-learnt code matrix $\mathbf{Z}$, i.e. formula (1), can be detailed as follows.

$$
\begin{aligned}
\begin{array}{l}
\Psi \\
=\max _{\mathbf{Z}} \operatorname{Tr}\left[\mathbf{Z}^{T} \mathbf{Y} \mathbf{Y}^{T} \mathbf{Z}\right]+\alpha \operatorname{Tr}\left[\mathbf{Z}^{T} \mathbf{H} \mathbf{Z}\right] \\
=\max _{\mathbf{Z}} \operatorname{Tr}\left[\mathbf{Z}^{T}\left(\mathbf{Y} \mathbf{Y}^{T}+\alpha \mathbf{H}\right) \mathbf{Z}\right]
\end{array} \\
\text { s.t. } \quad \mathbf{Z}^{T} \mathbf{Z}=\mathbf{I}
\end{aligned}
$$

where $\mathbf{H}=\mathbf{X}\left(\mathbf{X}^{T} \mathbf{X}\right)^{-1} \mathbf{X}^{T}$. As analysed in section IV, $\Psi$ can be transformed to an eigenvalue problem w.r.t $\mathbf{Y Y}^{T}+\alpha \mathbf{H}$, and $\mathbf{Z}$ is derived by concatenating the normalized eigenvectors corresponding to the top $d_{z}$ largest eigenvalues column by column. With the code matrix $\mathbf{Z}$ derived, predictive models can be trained for mapping instance features into code vectors.

4) Deriving Linear Decoding Matrix: According to formula (2) and (3), given $\mathbf{Z}$ with $\mathbf{Z}^{T} \mathbf{Z}=\mathbf{I}$, the optimal linear decoding matrix $\mathbf{Q}$ can be derived as follows.

$$
\mathbf{Q}=\left(\mathbf{Z}^{T} \mathbf{Z}\right)^{-1} \mathbf{Z}^{T} \mathbf{Y}=\mathbf{Z}^{T} \mathbf{Y}
$$

And its computational complexity is $\mathcal{O}\left(n d_{y} d_{z}\right)$.

An overview of $\mathrm{E}^{2} \mathrm{FE}$ is given in Algorithm 1.

\section{Error Analysis}

As shown in Algorithm 1, following PLST, CPLST and MLCSSP, the proposed $\mathrm{E}^{2} \mathrm{FE}$ rounds each entry of the decoding results into its nearest 0 and 1 , so as to derive binary label vectors. Considering that, we proceed to analyse the root mean square error (RMSE) of $\mathrm{E}^{2} \mathrm{FE}$ on the training instances.

Specifically, RMSE is defined as follows.

$$
R M S E=\frac{1}{\sqrt{n}}\|\operatorname{round}(\mathcal{G}(\mathbf{X}) \mathbf{Q})-\mathbf{Y}\|_{\text {fro }}
$$

where $\mathcal{G}$ denotes the learnt predictive models for mapping instance features into code vectors, and $\operatorname{round}(\mathcal{G}(\mathbf{X}) \mathbf{Q})$ denotes 
the recovered binary tagging matrix. Then we can derive the following lemma regarding the error bound of $\mathrm{E}^{2} \mathrm{FE}$.

Lemma 1. For $E^{2} F E$, its RMSE is bounded by

$$
R M S E \leq \frac{2}{\sqrt{n}}\left(\sqrt{d_{z}}\|\mathbf{Y}\|_{\text {fro }}\|\mathbf{Z}-\mathcal{G}(\mathbf{X})\|_{\text {fro }}+\|\mathbf{Y}-\mathbf{Z Q}\|_{\text {fro }}\right)
$$

For a detailed proof, one can refer to the supplementary material. Actually, the error bound for $\mathrm{E}^{2} \mathrm{FE}$ is similar to those of PLST and ML-CSSP. Namely, it also consists of two parts. The first part, i.e. $\sqrt{d_{z}}\|\mathbf{Y}\|_{\text {fro }}\|\mathbf{Z}-\mathcal{G}(\mathbf{X})\|_{\text {fro }}$ denotes the weighted training error of predictive models, and the second part, i.e. $\|\mathbf{Y}-\mathbf{Z Q}\|_{\text {fro }}$, denotes the loss of encoding label vectors into low-dimensional code vectors.

\section{Optimization Methods}

For optimizing the objective function $\Psi$ w.r.t the to-be-learnt code matrix $\mathbf{Z}$, any column $\mathbf{Z}_{\cdot, i}\left(i \in\left\{1,2, \ldots, d_{z}\right\}\right)$ can be derived with the following optimization sub-problem.

$$
\begin{aligned}
& \Psi^{(i)}=\max _{\mathbf{Z}, i} \mathbf{Z}_{\cdot, i}^{T}\left(\mathbf{Y} \mathbf{Y}^{T}+\alpha \mathbf{H}\right) \mathbf{Z} \cdot, i \\
& \text { s.t. } \quad \mathbf{Z}_{\cdot, i}^{T} \mathbf{Z}_{\cdot, i}=1, \mathbf{Z}_{\cdot, j}^{T} \mathbf{Z}_{\cdot, i}=0(\forall j<i)
\end{aligned}
$$

With the method of Lagrange multipliers, the optimal $\mathbf{Z}_{\cdot, i}$ should satisfy the following optimality condition.

$$
\left(\mathbf{Y} \mathbf{Y}^{T}+\alpha \mathbf{H}\right) \mathbf{Z}_{\cdot, i}=\lambda_{i} \mathbf{Z}_{\cdot, i}
$$

where $\lambda_{i}$ is the introduced Lagrange multiplier and will also be the optimal value of the sub-problem. It can be seen that the optimization for $\mathbf{Z}$ can be transformed to an eigenvalue problem. Then by normalizing the eigenvectors of $\mathbf{U}=$ $\mathbf{Y Y}^{T}+\alpha \mathbf{H}$ that correspond to the top $d_{z}$ largest eigenvalues, we can derive the optimal code matrix $\mathbf{Z}$ formed of these eigenvectors column by column, which satisfies $\mathbf{Z}^{T} \mathbf{Z}=\mathbf{I}$.

As described in our previous work [33], we can directly calculate $\mathbf{U}$ and then utilize effective methods to derive its eigenvectors. However, considering that $\mathbf{U} \in \mathbb{R}^{n \times n}$, for cases with $n \gg d_{x}+d_{y}$, which are common in practical applications, calculating $\mathbf{U}$ will result in high space costs. To avoid that, we derive the following lemma and further propose a more efficient optimization method for such cases.

Lemma 2. Given $\mathbf{H}=\mathbf{X}\left(\mathbf{X}^{T} \mathbf{X}\right)^{-1} \mathbf{X}^{T}$, the matrix $\mathbf{U}=$ $\mathbf{Y Y}^{T}+\alpha \mathbf{H}$ can be decomposed as $\mathbf{U}=\mathbf{V V}^{T}$ with $\mathbf{V} \in$ $\mathbb{R}^{n \times\left(d_{y}+d_{x}\right)}$. Also, the eigenvectors of $\mathbf{U}$ can be derived from those of $\mathbf{V}^{T} \mathbf{V}$, meaning that the size of the eigenvalue problem w.r.t $\mathbf{U}$ can be transformed from $\mathbb{R}^{n \times n}$ to $\mathbb{R}^{\left(d_{x}+d_{y}\right) \times\left(d_{x}+d_{y}\right)}$.

Proof. Suppose $\mathbf{A}=\mathbf{X}^{T} \mathbf{X}$ is invertible. Since $\mathbf{A} \in \mathbb{R}^{d_{x} \times d_{x}}$ is a real symmetric and positive-semidefinite matrix, $\mathbf{A}^{-1}$ will be real symmetric and positive semi-definite, and thus $\mathbf{A}^{-1}$ is diagonalizable by orthogonal matrices [41]. Namely, $\mathbf{A}^{-1}=$ $\mathbf{B} \boldsymbol{\Lambda} \mathbf{B}^{T}$, with $\boldsymbol{\Lambda}$ being a diagonal matrix having non-negative diagonal entries and $\mathbf{B}$ being an orthonormal matrix. Then $\mathbf{A}^{-1}=\mathbf{B} \boldsymbol{\Lambda}^{\frac{1}{2}} \boldsymbol{\Lambda}^{\frac{1}{2}} \mathbf{B}^{T}=\left(\mathbf{B} \boldsymbol{\Lambda}^{\frac{1}{2}}\right)\left(\mathbf{B} \boldsymbol{\Lambda}^{\frac{1}{2}}\right)^{T}$, where $\boldsymbol{\Lambda}^{\frac{1}{2}}$ is a diagonal matrix with each diagonal entry being the square root of the corresponding diagonal entry in $\boldsymbol{\Lambda}$. Furthermore, with $\mathbf{G}=\mathbf{X B} \Lambda^{\frac{1}{2}} \in \mathbb{R}^{n \times d_{x}}, \mathbf{H}=\mathbf{G G}^{T}$. Finally, $\mathbf{U}=\mathbf{Y} \mathbf{Y}^{T}+$

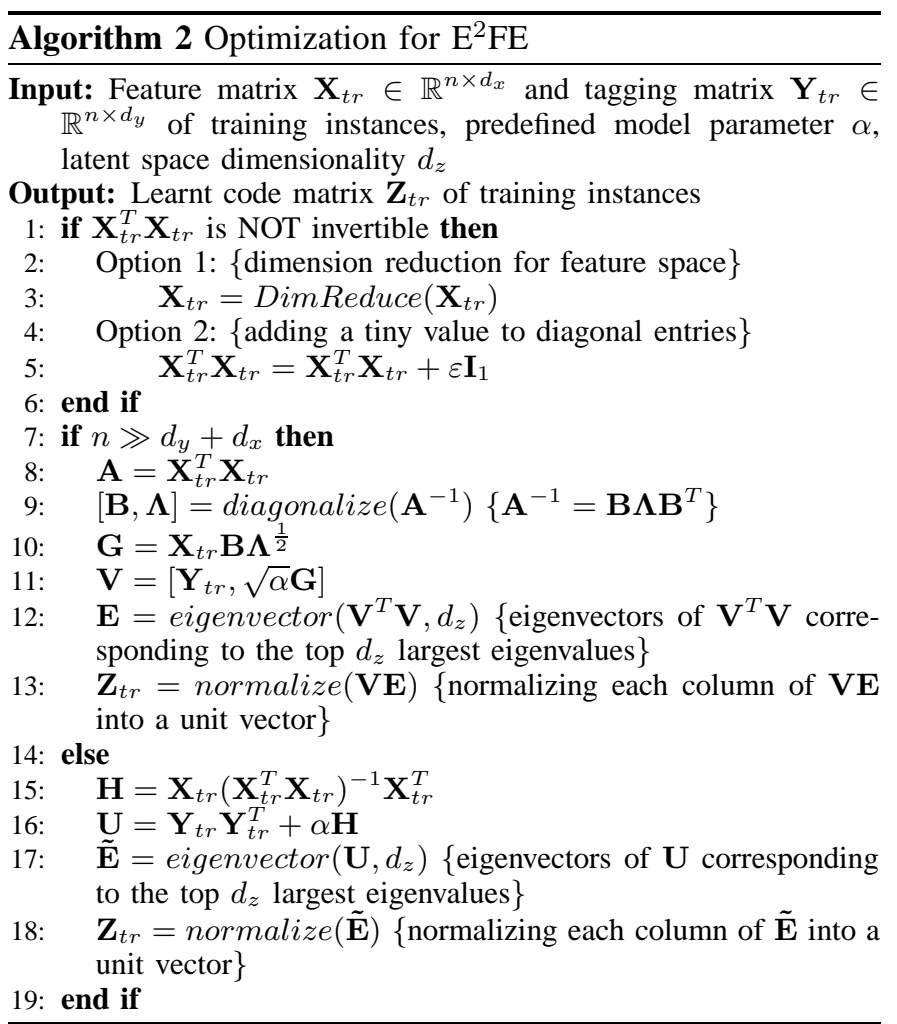

$\alpha \mathbf{H}=\mathbf{Y} \mathbf{Y}^{T}+(\sqrt{\alpha} \mathbf{G})(\sqrt{\alpha} \mathbf{G})^{T}=[\mathbf{Y}, \sqrt{\alpha} \mathbf{G}][\mathbf{Y}, \sqrt{\alpha} \mathbf{G}]^{T}=$ $\mathbf{V V}^{T}$, with $\mathbf{V}=[\mathbf{Y}, \sqrt{\alpha} \mathbf{G}] \in \mathbb{R}^{n \times\left(d_{y}+d_{x}\right)}$.

Suppose $\{\lambda, \mathbf{p}\}$ and $\{\sigma, \mathbf{q}\}$ are respectively the paired eigenvalue/eigenvector of $\mathbf{V} \mathbf{V}^{T}$ and $\mathbf{V}^{T} \mathbf{V}$. According to 1) $\mathbf{V} \mathbf{V}^{T} \mathbf{p}=\lambda \mathbf{p} \rightarrow\left(\mathbf{V}^{T} \mathbf{V}\right) \mathbf{V}^{T} \mathbf{p}=\mathbf{V}^{T}\left(\mathbf{V} \mathbf{V}^{T} \mathbf{p}\right)=\lambda \mathbf{V}^{T} \mathbf{p}$ and 2) $\mathbf{V}^{T} \mathbf{V q}=\sigma \mathbf{q} \rightarrow\left(\mathbf{V} \mathbf{V}^{T}\right) \mathbf{V q}=\mathbf{V}\left(\mathbf{V}^{T} \mathbf{V} \mathbf{q}\right)=\sigma \mathbf{V q}$, we can see that $\mathbf{V} \mathbf{V}^{T}$ and $\mathbf{V}^{T} \mathbf{V}$ share identical eigenvalues, and the eigenvectors of $\mathbf{U}=\mathbf{V V}^{T}$ can be derived from those of $\mathbf{V}^{T} \mathbf{V}$ based on the second derivation above. Considering $\mathbf{V}^{T} \mathbf{V} \in \mathbb{R}^{\left(d_{x}+d_{y}\right) \times\left(d_{x}+d_{y}\right)}$, the size of the eigenvalue problem w.r.t $\mathbf{U}$ can be transformed from $\mathbb{R}^{n \times n}$ to $\mathbb{R}^{\left(d_{x}+d_{y}\right) \times\left(d_{x}+d_{y}\right)}$.

With Lemma 2, in different cases we can utilize different optimization methods to obtain the eigenvectors of $\mathbf{U}=$ $\mathbf{Y Y}^{T}+\alpha \mathbf{H}$ and then derive the code matrix $\mathbf{Z}$, as summarized below and illustrated in Algorithm 2.

1) If $n \gg d_{y}+d_{x}$, it is preferable to firstly derive the matrix $\mathbf{V}$ satisfying $\mathbf{U}=\mathbf{V} \mathbf{V}^{T}$, then calculate the eigenvectors of $\mathbf{V}^{T} \mathbf{V}$ corresponding to the top $d_{z}$ largest eigenvalues, and finally utilize them to derive the eigenvectors of $\mathbf{U}$. Since $d_{z} \ll d_{y}$ and $\mathbf{V}^{T} \mathbf{V}$ is a real symmetric matrix, the eigenvalue problem w.r.t $\mathbf{V}^{T} \mathbf{V}$ can be solved efficiently using iterative methods like Arnoldi iteration [42], which can achieve an optimal computational complexity of $\mathcal{O}\left(d_{x} d_{z}{ }^{2}+d_{y} d_{z}{ }^{2}\right)$. Here the computational complexity of deriving $\mathbf{V}$ is $\mathcal{O}\left(n d_{x}^{2}\right)$, while that of calculating $\mathbf{V}^{T} \mathbf{V}$ and deriving the eigenvectors of $\mathbf{U}$ from those of $\mathbf{V}^{T} \mathbf{V}$ is $\mathcal{O}\left(n\left(d_{x}+d_{y}\right)^{2}\right)$.

2) Otherwise, it is preferable to directly calculate $\mathbf{U}$ and then perform an eigenvalue decomposition on it. The computational complexity for calculating $\mathbf{U}$ is at most 
$\mathcal{O}\left(\min \left\{n^{2} d_{x}, n d_{x}^{2}\right\}\right)+\mathcal{O}\left(n^{2} d_{x}+n^{2} d_{y}\right)$. Considering that generally $d_{z} \ll n$ and $\mathbf{U}$ is a real symmetric matrix, the eigenvalue problem w.r.t $\mathbf{U}$ can also be solved efficiently using Arnoldi iteration with an optimal computational complexity of $\mathcal{O}\left(n d_{z}{ }^{2}\right)$.

\section{V. $\pi \mathrm{E}^{2} \mathrm{FE}$ : Enhancing Linear Decoding Matrix with PRIORI KNOWLEDGE}

As analysed in formula (16), each column of the code matrix $\mathbf{Z}$ corresponds to an eigenvalue of $\mathbf{U}=\mathbf{Y} \mathbf{Y}^{T}+\alpha \mathbf{H}$, which is also the optimal value for its corresponding optimization sub-problem (i.e. formula (15)). Knowing that each column denotes one dimension of the latent space, for each column, the eigenvalue w.r.t it actually reflects 1 ) how predictable its corresponding dimension of the latent space is and 2) from the dimension how recoverable the label space is. Specifically, a higher eigenvalue w.r.t a column of $\mathbf{Z}$ means that its corresponding dimension of the latent space is more predictable and the label space is more recoverable from the dimension.

Here we propose to consider such priori knowledge to derive an enhanced linear decoding matrix for $\mathrm{E}^{2} \mathrm{FE}$. We denote it as $\pi \mathrm{E}^{2} \mathrm{FE}$. Essentially, for a linear decoding matrix $\mathbf{Q}$, its $i$ th column $\mathbf{Q}_{\cdot, i}\left(i \in\left\{1,2, \ldots, d_{y}\right\}\right)$ acts as a weighting vector to linearly combine dimensions of the latent space for recovering the $i$ th dimension of the label space. Then for dimensions of the latent space that are more predictable and make the label space more recoverable, i.e. with higher corresponding eigenvalues, they are expected to be assigned with higher weights in the decoding process. Therefore, we derive the objective function for $\mathbf{Q}_{., i}$ as follows.

$$
\tilde{\mathcal{L}}^{(i)}=\min _{\mathbf{Q}_{\cdot, i}}\left\|\mathbf{Y}_{\cdot, i}-\mathbf{Z Q} \mathbf{Q}_{\cdot, i}\right\|_{\text {fro }}^{2}-\eta \sum_{j=1}^{d_{z}} \lambda_{j} \mathbf{Q}_{j, i}^{2}
$$

where $\mathbf{Y}_{\cdot, i}$ is the $i$ th column of the tagging matrix $\mathbf{Y}, \lambda_{j}$ is the eigenvalue corresponding to the $j$ th column of $\mathbf{Z}$ and $\eta$ is a non-negative weighting factor. It can be seen that, by considering the priori knowledge as a regularizer in $\tilde{\mathcal{L}}^{(i)}$, a larger $\lambda_{j}$ can help to lead $\mathbf{Q}_{j, i}^{2}$ to be larger, meaning that as expected the $j$ th dimension of the latent space is assigned with a higher weight for decoding. For model simplicity, here $\eta$ is shared by all $\mathbf{Q}_{\cdot, i}\left(i \in\left\{1,2, \ldots, d_{y}\right\}\right)$. Then the objective function for deriving the linear decoding matrix $\mathbf{Q}$ of $\pi \mathrm{E}^{2} \mathrm{FE}$ can be formulated as follows with matrix notations.

$$
\tilde{\mathcal{L}}=\min _{\mathbf{Q}}\|\mathbf{Y}-\mathbf{Z Q}\|_{\text {fro }}^{2}-\eta \operatorname{Tr}\left[\mathbf{Q}^{T} \tilde{\mathbf{\Lambda}} \mathbf{Q}\right]
$$

where $\tilde{\Lambda}$ is a diagonal matrix with $\tilde{\Lambda}_{j, j}=\lambda_{j}$. If $\eta$ is properly set to make $\tilde{\mathcal{L}}$ non-trivial, as discussed later, the optimal decoding matrix for $\pi \mathrm{E}^{2} \mathrm{FE}$ can be derived as follows.

$$
\mathbf{Q}=(\mathbf{I}-\eta \tilde{\mathbf{\Lambda}})^{-1} \mathbf{Z}^{T} \mathbf{Y}
$$

where $(\mathbf{I}-\eta \tilde{\mathbf{\Lambda}})$ is a diagonal matrix and thus its inverse can be efficiently calculated. Actually, as $d_{z} \ll d_{y}$, the computational complexity of deriving $\mathbf{Q}$ in $\pi \mathrm{E}^{2} \mathrm{FE}$ is also $\mathcal{O}\left(n d_{y} d_{z}\right)$.

Note that in formula (18), a large $\eta$ can lead $\tilde{\mathcal{L}}$ to become trivial and achieve an optimum of negative infinity. To cope with that, we derive the following lemma for properly setting $\eta$, where $\vec{\lambda}=\left[\lambda_{1}, \lambda_{2}, \ldots, \lambda_{d_{z}}\right]$.

Lemma 3. For any $\eta \in\left[0, \frac{1}{\max (\vec{\lambda})}\right]$ with $\max (\vec{\lambda})$ being the maximal value of $\vec{\lambda}, \tilde{\mathcal{L}}$ will be non-trivial for optimization.

For a detailed proof, one can refer to the supplementary material.

\section{EXtensions AND ANALYSES}

\section{A. Function-based Encoding: a Linear Encoding Case}

Though the proposed $\mathrm{E}^{2} \mathrm{FE}$ requires no encoding function, it can still be specified to learn an encoding function as most previous works, given that the encoding function can be optimized, e.g. a linear one, as described below.

Following PLST and CPLST, we use an encoding matrix $\mathbf{P} \in \mathbb{R}^{d_{y} \times d_{z}}$ to denote the linear encoding function. Then the code matrix $\mathbf{Z}$ can be expressed as $\mathbf{Z}=\mathbf{Y} \mathbf{P}$. Substituting $\mathbf{Z}$ with $\mathrm{YP}$ in the objective function of $\mathrm{E}^{2} \mathrm{FE}$, i.e. formula (12), we can derive the following objective function for $\mathbf{P}$.

$$
\begin{aligned}
& \Psi=\max _{\mathbf{P}} \operatorname{Tr}\left[\mathbf{P}^{T}\left(\mathbf{Y}^{T} \mathbf{Y} \mathbf{Y}^{T} \mathbf{Y}+\alpha \mathbf{Y}^{T} \mathbf{H Y}\right) \mathbf{P}\right] \\
& \text { s.t. } \quad \mathbf{P}^{T} \mathbf{Y}^{T} \mathbf{Y} \mathbf{P}=\mathbf{I}
\end{aligned}
$$

Similarly, we use the method of Lagrange multipliers and decompose $\Psi$ into $d_{z}$ optimization sub-problems w.r.t each column $\mathbf{P}_{\cdot, i}$ of the to-be-learnt $\mathbf{P}$. Then we derive that $\mathbf{P}_{\cdot, i}$ should satisfy the following optimality condition.

$$
\left(\mathbf{Y}^{T} \mathbf{Y} \mathbf{Y}^{T} \mathbf{Y}+\alpha \mathbf{Y}^{T} \mathbf{H Y}\right) \mathbf{P}_{\cdot, i}=\lambda_{i}\left(\mathbf{Y}^{T} \mathbf{Y}\right) \mathbf{P}_{\cdot, i}
$$

where $\lambda_{i}$ is a Lagrange multiplier and will be the optimal value of the optimization sub-problem w.r.t $\mathbf{P}_{\cdot, i}$. It can be seen that the optimization of $\mathbf{P}$ is essentially a general eigenvalue problem. And the normalized eigenvectors corresponding to the top $d_{z}$ largest eigenvalues will form the optimal $\mathbf{P}$.

Denoting this case of linear function-based encoding as LinearE ${ }^{2} \mathrm{FE}$, the linear decoding matrix $\mathbf{Q}$ without considering priori knowledge is $\mathbf{Q}=(\mathbf{Y} \mathbf{P})^{T} \mathbf{Y}$. Meanwhile, for the case of utilizing the eigenvalues w.r.t $\mathbf{P}$ as priori knowledge, $\mathbf{Q}=(\mathbf{I}-\eta \tilde{\mathbf{\Lambda}})^{-1}(\mathbf{Y} \mathbf{P})^{T} \mathbf{Y}$ with $\tilde{\mathbf{\Lambda}}$ being a diagonal matrix consisting of the eigenvalues, which is termed $\pi$ LinearE $^{2} \mathrm{FE}$.

\section{B. Kernel Version}

The proposed $\mathrm{E}^{2} \mathrm{FE}$, thanks to kernel tricks, can be extended to deal with non-linear correlations between the feature space and the latent space, which is termed kernel- $\mathrm{E}^{2} \mathrm{FE}$.

In kernel- $\mathrm{E}^{2} \mathrm{FE}$, each feature vector $\mathrm{x}^{(i)}$ is mapped to the Reproducing Kernel Hilbert Space (RKHS) as $\phi\left(\mathbf{x}^{(i)}\right)$. In RKHS, the inner product between $\phi\left(\mathbf{x}^{(i)}\right)$ and $\phi\left(\mathbf{x}^{(j)}\right)$ is equal to $\kappa\left(\mathbf{x}^{(i)}, \mathbf{x}^{(j)}\right)$, where $\kappa(\cdot, \cdot)$ is the introduced kernel function. Using a non-linear $\kappa(\cdot, \cdot)$, the linear correlations between the RKHS and the latent space actually reflect the non-linear correlations between the original feature space and the latent space. Similar to formula (7), we measure the correlation $r(\boldsymbol{\Phi}, \mathbf{z})$ by considering a linear projection $\mathbf{w}_{1}$ for kernel features in RKHS and a column $\mathbf{z}$ of the code matrix. Following [43], here $\mathbf{w}_{1}$ is assumed to be in the span of 
sampled kernel feature vectors, i.e. $\mathbf{w}_{1}=\boldsymbol{\Phi}_{*}^{T} \tilde{\mathbf{w}}$ where $\boldsymbol{\Phi}_{*}$ is a matrix built by the sampled kernel feature vectors row by row and $\tilde{\mathbf{w}}$ is an $s$-dimensional weighting vector with $s$ being the sampling size. Then $r(\boldsymbol{\Phi}, \mathbf{z})$ can be measured as follows.

$$
\begin{aligned}
r(\boldsymbol{\Phi}, \mathbf{z}) & =\frac{\left(\boldsymbol{\Phi} \boldsymbol{\Phi}_{*}^{T} \tilde{\mathbf{w}}\right)^{T} \mathbf{z}}{\sqrt{\left(\boldsymbol{\Phi} \boldsymbol{\Phi}_{*}^{T} \tilde{\mathbf{w}}\right)^{T}\left(\boldsymbol{\Phi} \boldsymbol{\Phi}_{*}^{T} \tilde{\mathbf{w}}\right)} \sqrt{\mathbf{z}^{T} \mathbf{z}}} \\
& =\frac{(\mathbf{K} \tilde{\mathbf{w}})^{T} \mathbf{z}}{\sqrt{(\mathbf{K} \tilde{\mathbf{w}})^{T}(\mathbf{K} \tilde{\mathbf{w}})} \sqrt{\mathbf{z}^{T} \mathbf{z}}}
\end{aligned}
$$

where $\mathbf{K}=\boldsymbol{\Phi} \boldsymbol{\Phi}_{*}^{T} \in \mathbb{R}^{n \times s}$ is a kernel matrix and can be efficiently derived using the kernel function with the original feature vectors. Similar to subsection III-B2, the predictability of $\mathbf{z}$ based on non-linear correlations can be derived from the maximal $r(\boldsymbol{\Phi}, \mathbf{z})$ and measured as $\psi_{2}(\boldsymbol{\Phi}, \mathbf{z})=\sqrt{\mathbf{z}^{T} \tilde{\mathbf{H}} \mathbf{z}}$ with $\tilde{\mathbf{H}}=\mathbf{K}\left(\mathbf{K}^{T} \mathbf{K}\right)^{-1} \mathbf{K}^{T}$. Then the objective function of kernel- $\mathrm{E}^{2} \mathrm{FE}$ is as follows, which can also be transformed to an eigenvalue problem.

$$
\Psi=\max _{\mathbf{Z}} \operatorname{Tr}\left[\mathbf{Z}^{T}\left(\mathbf{Y} \mathbf{Y}^{T}+\alpha \tilde{\mathbf{H}}\right) \mathbf{Z}\right] \quad \text { s.t. } \quad \mathbf{Z}^{T} \mathbf{Z}=\mathbf{I}
$$

Like $\mathrm{E}^{2} \mathrm{FE}$, the linear decoding matrix $\mathbf{Q}$ for kernel- $\mathrm{E}^{2} \mathrm{FE}$ is $\mathbf{Q}=\mathbf{Z}^{T} \mathbf{Y}$. Meanwhile, when eigenvalues w.r.t $\mathbf{Z}$ are considered as priori knowledge, $\mathbf{Q}=(\mathbf{I}-\eta \tilde{\mathbf{\Lambda}})^{-1} \mathbf{Z}^{T} \mathbf{Y}$, where $\tilde{\Lambda}$ is a diagonal matrix consisting of eigenvalues. Here we denote this case as kernel- $\pi \mathrm{E}^{2} \mathrm{FE}$.

\section{Relations to Previous Works}

If the mean values of the label vectors are shifted as zeros, the proposed $\mathrm{E}^{2} \mathrm{FE}$ will degenerate to PLST [28] when only the recoverability of the label space is considered (i.e. $\alpha=0$ in formula (12)). Here, we denote this case as R-E $\mathrm{E}^{2} \mathrm{FE}$ and its corresponding objective function is given as follows.

$$
\Psi=\max _{\mathbf{Z}} \operatorname{Tr}\left[\mathbf{Z}^{T} \mathbf{Y} \mathbf{Y}^{T} \mathbf{Z}\right], \quad \text { s.t. } \quad \mathbf{Z}^{T} \mathbf{Z}=\mathbf{I}
$$

The code matrix $\mathbf{Z}$ consists of the normalized eigenvectors of $\mathbf{Y Y}^{T}$ corresponding to the top $d_{z}$ largest eigenvalues, and the linear decoding matrix without considering priori knowledge is $\mathbf{Z}^{T} \mathbf{Y}$. Meanwhile, the linear encoding matrix $\mathbf{P}$ of PLST is formed with normalized eigenvectors of $\mathbf{Y}^{T} \mathbf{Y}$ corresponding to the top $d_{z}$ largest eigenvalues, with the derived code matrix being $\mathbf{Y} \mathbf{P}$ and the linear decoding matrix being $\mathbf{P}^{T}$. As in the proof of Lemma 2, we can derive that $\mathbf{Y} \mathbf{Y}^{T}$ and $\mathbf{Y}^{T} \mathbf{Y}$ are positive semi-definite and share the same positive eigenvalues. Specifically, provided that $\lambda_{i}$ is the $i$ th largest eigenvalue, we can derive that: 1) $\mathbf{Y}^{T} \mathbf{Y} \mathbf{P}_{\cdot, i}=\lambda_{i} \mathbf{P}_{\cdot, i}$;2) $\mathbf{Y} \mathbf{Y}^{T} \mathbf{Z}_{\cdot, i}=$ $\lambda_{i} \mathbf{Z}_{\cdot, i}$; 3) $\left(\mathbf{Y Y}^{T}\right)[\mathbf{Y P}]_{\cdot, i}=\mathbf{Y}\left(\mathbf{Y}^{T} \mathbf{Y} \mathbf{P}_{\cdot, i}\right)=\lambda_{i}[\mathbf{Y P}]_{,, i}$; 4) $\left(\mathbf{Y}^{T} \mathbf{Y}\right)\left[\mathbf{Y}^{T} \mathbf{Z}\right]_{\cdot, i}=\mathbf{Y}^{T}\left(\mathbf{Y Y}^{T} \mathbf{Z} \cdot, i\right)=\lambda_{i}\left[\mathbf{Y}^{T} \mathbf{Z}\right]_{\cdot, i}$. Then for $\mathrm{R}-\mathrm{E}^{2} \mathrm{FE}$ and PLST, we can find one-to-one correspondences between the $i$ th columns of their encoding results (i.e. $\mathbf{Z}_{\cdot, i}=$ $\left.\frac{[\mathbf{Y P}]_{, i}}{\sqrt{\lambda_{i}}}\right)$, and between the $i$ th rows of their linear decoding matrices (i.e. $\left[\mathbf{Z}^{T} \mathbf{Y}\right]_{i, .}=\sqrt{\lambda_{i}}\left[\mathbf{P}^{T}\right]_{i,}$ ). Therefore, $\mathrm{R}-\mathrm{E}^{2} \mathrm{FE}$ is equivalent to PLST, with $\mathbf{Z}\left(\mathbf{Z}^{T} \mathbf{Y}\right)=(\mathbf{Y} \mathbf{P}) \mathbf{P}^{T}$. However, when $\alpha>0$, the code matrix $\mathbf{Z}$ in $\mathrm{E}^{2} \mathrm{FE}$ will be associated to instance features and will then differ from PLST.

When coping with linear function-based encoding, i.e. formula (20), given the mean values of label vectors and those of feature vectors shifted as zeros, $\mathrm{E}^{2} \mathrm{FE}$ is closely connected to CPLST [30] if only the predictability of the latent space is considered. We denote this case as P-LinearE ${ }^{2} \mathrm{FE}$, with its corresponding objective function defined as follows.

$$
\Psi=\max _{\mathbf{P}} \operatorname{Tr}\left[\mathbf{P}^{T} \mathbf{Y}^{T} \mathbf{H Y} \mathbf{P}\right] \text {, s.t. } \mathbf{P}^{T} \mathbf{Y}^{T} \mathbf{Y} \mathbf{P}=\mathbf{I}
$$

Meanwhile, the objective function of CPLST is as follows.

$$
\tilde{\Psi}=\max _{\mathbf{P}} \operatorname{Tr}\left[\mathbf{P}^{T} \mathbf{Y}^{T} \mathbf{H Y P}\right], \quad \text { s.t. } \quad \mathbf{P}^{T} \mathbf{P}=\mathbf{I}
$$

It can be seen that P-LinearE ${ }^{2} \mathrm{FE}$ and CPLST share an identical objective function but with different constraints. Namely, the former requires dimensions of the code matrix (i.e. YP) to be orthonormal while the latter requires dimensions of the linear encoding matrix (i.e. $\mathbf{P}$ ) to be orthonormal.

Another useful observation w.r.t $\mathrm{E}^{2} \mathrm{FE}$ is that $\mathrm{E}^{2} \mathrm{FE}$ actually performs dimensionality reduction for both the label space and the feature space when the predictability of the latent space is over-emphasized with an assumption that the code matrix can be directly expressed by the feature matrix, i.e. $\mathbf{Z}=\mathbf{X W}$ where $\mathbf{W} \in \mathbb{R}^{d_{x} \times d_{z}}$ is a regression matrix. This case is termed $\mathrm{OP}-\mathrm{E}^{2} \mathrm{FE}$. As the predictability of the latent space is constant in OP-E ${ }^{2} \mathrm{FE}$, its objective function is formulated as follows.

$$
\begin{aligned}
& \Psi=\max _{\mathbf{W}} \operatorname{Tr}\left[\mathbf{W}^{T} \mathbf{X}^{T} \mathbf{Y} \mathbf{Y}^{T} \mathbf{X W}\right] \\
& \text { s.t. } \quad \mathbf{W}^{T} \mathbf{X}^{T} \mathbf{X W}=\mathbf{I}
\end{aligned}
$$

The optimization for $\mathbf{W}$ can again be interpreted as a general eigenvalue problem, i.e. $\left(\mathbf{X}^{T} \mathbf{Y} \mathbf{Y}^{T} \mathbf{X}\right) \mathbf{W}_{\cdot, i}=\lambda_{i}\left(\mathbf{X}^{T} \mathbf{X}\right) \mathbf{W}_{\cdot, i}$, but it requires $d_{z} \leq d_{x}$. Here $\mathbf{Z}$ can be seen as the dimensionality reduction result learnt from the label space in an end-to-end manner, or the linear dimensionality reduction result from the feature space with W. However, for OP-E ${ }^{2} \mathrm{FE}$, we can observe the following weak points. 1) The dimensionality of the tobe-learnt latent space cannot be larger than the dimensionality of the feature space, which can sometimes be too small to keep enough information of the label space, especially when $d_{x} \ll d_{y}$. 2) The predictive models from the feature space to the latent space are limited to be linear regression whereas for LSDR they are expected to be open for any effective model.

\section{EXPERIMENTS}

\section{A. Experimental Settings}

To validate the proposed $\mathrm{E}^{2} \mathrm{FE}$, we use in our experiments five widely-used benchmark datasets with relatively large vocabularies from Mulan [44], i.e. delicious, CAL500, mediamill, bibtex and bookmarks. They belong to one of the following domains: text, music and video. Moreover, following CS [27], we also conduct experiments on the image dataset ESPGame [45], and take those tags appearing at least 20 times in the dataset to form a large vocabulary, which almost doubles the size of that used in the experiments of CS. Each instance in ESPGame is represented by a 516-D feature vector ${ }^{1}$ extracted with Lire [46], and it is removed if no tags are associated. The original statistics of the datasets are given in Table III.

For performance comparison, we select Binary Relevance (BR) [47], CS [27], PLST [28], CPLST and kernel-CPLST

1516-D feature vector: 60-D Gabor, 192-D FCTH, 80-D Edge Histogram, 120-D Color Layout and 64-D RGB Color Histogram 
TABLE III

STATISTICS OF DATASETS

\begin{tabular}{l|c|c|c|c} 
& domain & instances & labels & features \\
\hline \hline delicious & text & 16,105 & 983 & 500 \\
CAL500 & music & 502 & 174 & 68 \\
mediamill & video & 43,907 & 101 & 120 \\
ESPGame & image & 65,065 & 1,718 & 516 \\
bibtex & text & 7,395 & 159 & 1,836 \\
bookmarks & text & 87,856 & 208 & 2,150
\end{tabular}

[30], MLC-BMaD [31] and ML-CSSP [32] as baselines, where $\mathrm{BR}$ is a widely-used multi-label classification method that trains a separate binary relevance model for each label. In our experiments, we use both linear SVM (L-SVM) [48] and linear ridge regression (L-RR) for $\mathrm{BR}$. And for the latter, we use 0.5 as a threshold to decide the binary ( 0 or 1$)$ classification results. To reduce the computational costs of L-SVM on bibtex and bookmarks, we perform feature dimensionality reduction for both datasets via PCA. We also follow the reported preprocessing steps of baselines, like shifting the mean values of feature vectors to be zeros, etc. Note that BR in fact does not perform LSDR and thus its performance is a reference for other algorithms. BML-CS [5] is not included since it is sophisticated with numerous parameters to tune.

For $\mathrm{E}^{2} \mathrm{FE}$, we evaluate the following variants. 1) $\mathrm{R}-\mathrm{E}^{2} \mathrm{FE}$ : considering only the recoverability of the label space (i.e. formula (24)), theoretically equivalent to PLST; 2) P-LinearE ${ }^{2} \mathrm{FE}$ : considering only the predictability of the latent space for linear function-based encoding (i.e. formula (25)), similar to CPLST; 3) OP-E ${ }^{2} \mathrm{FE}$ : over-emphasizing the predictability of the latent space (i.e. formula (27)); 4) LinearE ${ }^{2} \mathrm{FE}$ : linear function-based encoding (i.e. formula (20)); 5) $\pi$ LinearE $^{2} \mathrm{FE}$ : identical to LinearE ${ }^{2} \mathrm{FE}$ except that the linear decoding matrix is learnt with priori knowledge; 6) $\mathrm{E}^{2} \mathrm{FE}$ : end-to-end feature-aware label space encoding (i.e. formula (12)); 7) $\pi \mathrm{E}^{2} \mathrm{FE}$ : identical to $\mathrm{E}^{2} \mathrm{FE}$ except that the linear decoding matrix is learnt with priori knowledge; 8) kernel- $\mathrm{E}^{2} \mathrm{FE}$ : kernel version of $\mathrm{E}^{2} \mathrm{FE}$ (i.e. formula (23)); 9) kernel- $\pi \mathrm{E}^{2} \mathrm{FE}$ : identical to kernel- $\mathrm{E}^{2} \mathrm{FE}$ except that the linear decoding matrix is learnt with priori knowledge.

In our experiments, each dataset is evenly and randomly divided into 5 parts. Five runs of each algorithm are then performed on the dataset, taking each time one part for testing and the rest for training without duplication. Experimental results are measured with widely-used metrics in the field of multilabel classification, i.e. label-based macroFl and examplebased Accuracy [49], and then averaged over the 5 runs. Higher label-based macroFl and example-based Accuracy means better performance. Specifically, for each run, labelbased macroFl is calculated as follows.

$$
\begin{aligned}
& \text { macroF } 1=\frac{1}{d_{y}} \sum_{i=1}^{d_{y}} \frac{2 p_{i} r_{i}}{p_{i}+r_{i}} \\
& \text { s.t. } \quad p_{i}=\frac{\left|G_{i} \cap P_{i}\right|}{\left|P_{i}\right|}, r_{i}=\frac{\left|G_{i} \cap P_{i}\right|}{\left|G_{i}\right|}
\end{aligned}
$$

where $d_{y}$ is the number of all labels, $G_{i}$ and $P_{i}$ are respectively the sets of the ground-truth and the predicted positive instances for the $i$ th label, and $\cap, \cup$ are operations of intersection and union between two sets. Meanwhile, example-based Accuracy is given by the following formula.

$$
\text { Accuracy }=\frac{1}{n_{t}} \sum_{j=1}^{n_{t}} \frac{\left|G_{j}^{\prime} \cap P_{j}^{\prime}\right|}{\left|G_{j}^{\prime} \cup P_{j}^{\prime}\right|}
$$

where $n_{t}$ is the test set size, $G_{j}^{\prime}$ and $P_{j}^{\prime}$ are respectively the ground-truth and the predicted label set of the $j$ th test instance.

Moreover, for each run of any algorithm, we conduct 5fold cross-validation on the training set for selecting model parameters via grid search in predefined value ranges. Specifically, $\alpha$ in the proposed $\mathrm{E}^{2} \mathrm{FE}$ and its variants is selected from $\left\{10^{-1}, 10^{0}, \ldots, 10^{4}\right\}, \tau$ for MLC-BMaD is chosen from $\{0.1,0.2, \ldots, 1.0\}$, and the predefined sparsity level in CS is selected from $\{1,2, \ldots, M\}$ with $M$ being the maximal number of labels in an instance, etc. Additionally, for $\eta$ in $\pi \mathrm{E}^{2} \mathrm{FE} / \pi$ LinearE $\mathrm{E}^{2} \mathrm{FE} /$ kernel $-\pi \mathrm{E}^{2} \mathrm{FE}$, we set $\eta=\xi \frac{1}{\max (\vec{\lambda})}$ for each dataset and select $\xi$ from $\left\{0,2^{-10}, 2^{-9}, \ldots, 2^{-1}, 1\right\}$ via cross-validation. Following most previous works, like [5], [28], [30], [32], we utilize linear ridge regression as predictive models to learn the mappings from instance features to code vectors. As for kernel-CPLST, kernel- $\mathrm{E}^{2} \mathrm{FE}$ and kernel- $\pi \mathrm{E}^{2} \mathrm{FE}$, we empirically utilize the Gaussian kernel function and set the smoothing parameter $\sigma$ as twice the mean Euclidean distance between feature vectors for each dataset. Accordingly, we utilize kernel ridge regression as predictive models for them to learn the non-linear mappings from instance features to code vectors. Moreover, following PLST, CPLST and ML-CSSP, we round each continuous entry of the decoding results into its nearest 0 or 1 to get the binary label vectors for test instances.

\section{B. Experimental Results of LSDR}

We run all algorithms on the six datasets with different values of $d_{z} / d_{y}$ (mostly from $10 \%$ to $50 \%$ ) where $d_{z}$ and $d_{y}$ are respectively the dimensionality of the latent space and that of the label space. Particularly, for ESPGame, $d_{z} / d_{y}$ is varied from $5 \%$ to $25 \%$, as it has a much larger vocabulary.

1) Performance Comparison with Baselines: The experimental results of compared baselines and variants of the proposed $\mathrm{E}^{2} \mathrm{FE}$ are reported in Table IV and $\mathrm{V}$.

A close look at the achieved results reveals: 1) The proposed $\mathrm{E}^{2} \mathrm{FE}$ as well as its linear function-based variant LinearE ${ }^{2} \mathrm{FE}$ generally outperform the compared baselines on each dataset, which clearly demonstrates their effectiveness. 2) $\mathrm{E}^{2} \mathrm{FE}$ outperforms LinearE ${ }^{2} \mathrm{FE}$ on all datasets, reflecting the superiority of learning code vectors in an end-to-end manner rather than a function-based manner. 3) $\mathrm{E}^{2} \mathrm{FE}$ outperforms R-E ${ }^{2} \mathrm{FE}$ and LinearE ${ }^{2} \mathrm{FE}$ outperforms P-LinearE ${ }^{2} \mathrm{FE}$, which implies that jointly considering predictability and recoverability will obtain better performance. 4) $\mathrm{OP}-\mathrm{E}^{2} \mathrm{FE}$ yields inferior performance to $\mathrm{E}^{2} \mathrm{FE}$ and cannot even perform LSDR on CAL500 when $d_{z} / d_{y} \geq 40 \%$, as the dimensionality of the feature space will be smaller than $d_{z}$. That points out the weakness of OP$\mathrm{E}^{2} \mathrm{FE}$ and further validates the superiority of keeping a good trade-off between predictability and recoverability. 5) R-E ${ }^{2} \mathrm{FE}$ yields nearly the same performance as PLST, as predicted by our theoretical analyses about their equivalence. 6) With an identical objective function but different orthogonality constraints, P-LinearE ${ }^{2} \mathrm{FE}$ seems to be slightly superior to 
1

2

3

4

5

6

7

8

9

TABLE IV

EXPERIMENTAL RESULTS: label-based macroF1 ON delicious, CAL500, mediamill, ESPGame, bibtex AND bookmarks, WITH VARYING $d_{z} / d_{y}$

\begin{tabular}{|c|c|c|c|c|c|c|c|c|c|c|c|c|c|c|c|}
\hline \multirow{2}{*}{$\begin{array}{l}\text { Datasets } \\
d_{z} / d_{y} \\
\end{array}$} & \multicolumn{5}{|c|}{ delicious } & \multicolumn{5}{|c|}{ CAL500 } & \multicolumn{5}{|c|}{ mediamill } \\
\hline & $10 \%$ & $20 \%$ & $30 \%$ & $40 \%$ & $50 \%$ & $10 \%$ & $20 \%$ & $30 \%$ & $40 \%$ & $50 \%$ & $10 \%$ & $20 \%$ & $30 \%$ & $40 \%$ & $50 \%$ \\
\hline L-SVM & \multirow{2}{*}{\multicolumn{5}{|c|}{$\begin{array}{l}0.0951 \\
0.0377\end{array}$}} & \multirow{2}{*}{\multicolumn{5}{|c|}{$\begin{array}{l}0.1397 \\
0.0569\end{array}$}} & \multirow{2}{*}{\multicolumn{5}{|c|}{$\begin{array}{l}0.0866 \\
0.0447\end{array}$}} \\
\hline L-RR & & & & & & & & & & & & & & & \\
\hline $\begin{array}{c}\text { CS [27] } \\
\text { PLST [28] }\end{array}$ & 0.0063 & 0.0208 & 0.0422 & 0.0466 & 0.0415 & 0.0677 & 0.0820 & 0.0906 & 0.0976 & 0.1142 & 0.0052 & 0.0144 & 0.0138 & 0.0319 & 0.0304 \\
\hline PLST [28] & 0.0234 & 0.0256 & 0.0271 & 0.0278 & 0.0284 & 0.0604 & 0.0605 & 0.0606 & 0.0609 & 0.0608 & 0.0422 & 0.0439 & 0.0448 & 0.0447 & 0.0447 \\
\hline CPLST [30] & 0.0339 & 0.0341 & 0.0341 & 0.0341 & 0.0341 & 0.0640 & 0.0643 & 0.0644 & 0.0645 & 0.0645 & 0.0432 & 0.0446 & 0.0447 & 0.0447 & 0.0447 \\
\hline \multirow{2}{*}{$\begin{array}{c}\text { MLC-BMaD [31] } \\
\text { ML-CSSP [32] }\end{array}$} & 0.0238 & 0.0259 & 0.0297 & 0.0344 & 0.0347 & 0.0485 & 0.0444 & 0.0420 & 0.0472 & 0.0468 & 0.0398 & 0.0408 & 0.0408 & 0.0408 & 0.0408 \\
\hline & 0.0160 & 0.0216 & 0.0277 & 0.0324 & 0.0319 & 0.0453 & 0.0498 & 0.0507 & 0.0528 & 0.0543 & 0.0354 & 0.0395 & 0.0426 & 0.0433 & 0.0427 \\
\hline 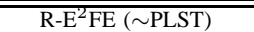 & $\overline{0.0234}$ & $\overline{0.0257}$ & 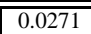 & $\overline{0.0278}$ & $\overline{0.0285}$ & $\overline{0.0592}$ & $\overline{0.0590}$ & 0.0592 & $\overline{0.0593}$ & $\overline{0.0593}$ & $\overline{0.0422}$ & $\overline{0.0439}$ & $\overline{c 0.0448}$ & "0.0447 & $\overline{0.0447}$ \\
\hline P-LinearE ${ }^{2} \mathrm{FE}(\sim \mathrm{CPLST})$ & 0.0391 & 0.0398 & 0.0399 & 0.0399 & 0.0400 & 0.0795 & 0.0954 & 0.1008 & 0.1003 & 0.1003 & 0.0420 & 0.0437 & 0.0446 & 0.0446 & 0.0447 \\
\hline \multirow{2}{*}{$\frac{\mathrm{OP}-\mathrm{E}^{2} \mathrm{FE}}{\text { LinearE }^{2} \mathrm{FE}}$} & 0.0449 & 0.0470 & 0.0476 & 0.0478 & 0.0475 & 0.1034 & 0.1080 & 0.1088 & & & 0.0433 & 0.0447 & 0.0448 & 0.0449 & 0.0448 \\
\hline & 0.0413 & 0.0417 & 0.0416 & 0.0416 & 0.0416 & 0.1061 & 0.1115 & 0.1110 & 0.1101 & 0.1101 & 0.0440 & 0.0449 & 0.0451 & 0.0449 & 0.0448 \\
\hline $\mathrm{E}^{2} \mathrm{FE}$ & 0.0530 & 0.0569 & 0.0577 & 0.0578 & 0.0578 & 0.1198 & 0.1247 & 0.1263 & 0.1258 & 0.1256 & 0.0549 & 0.0575 & 0.0577 & 0.0577 & 0.0577 \\
\hline \multirow{2}{*}{$\begin{array}{c}\text { kernel-CPLST [30] } \\
\text { kernel-E }{ }^{2} \mathrm{FE}\end{array}$} & 0.0354 & 0.0377 & 0.0383 & 0.0389 & 0.0393 & 0.0754 & 0.0774 & 0.0774 & 0.0774 & 0.0774 & 0.0594 & 0.0688 & 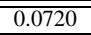 & 0.0751 & 0.0756 \\
\hline & 0.0500 & 0.0569 & 0.0591 & 0.0599 & 0.0599 & 0.1160 & 0.1208 & 0.1215 & 0.1272 & 0.1307 & 0.0692 & 0.0814 & 0.0945 & 0.0997 & 0.1003 \\
\hline \multirow{2}{*}{$\begin{array}{c}\text { Datasets } \\
d_{z} / d_{y} \\
\end{array}$} & \multicolumn{5}{|c|}{ ESPGame } & \multicolumn{5}{|c|}{ bibtex } & \multicolumn{5}{|c|}{ bookmarks } \\
\hline & $5 \%$ & $10 \%$ & & $20 \%$ & $25 \%$ & $10 \%$ & $20 \%$ & $30 \%$ & $40 \%$ & $50 \%$ & $10 \%$ & $20 \%$ & $30 \%$ & $40 \%$ & $50 \%$ \\
\hline \multirow{3}{*}{\begin{tabular}{c|r} 
BR [47] & L- \\
\cline { 2 - 2 } & CS [27] \\
\multicolumn{2}{|c|}{} \\
\end{tabular}} & \multirow{2}{*}{\multicolumn{5}{|c|}{0.0688}} & \multirow{2}{*}{\multicolumn{5}{|c|}{$\begin{array}{ll}0.3023 \\
0.0613\end{array}$}} & \multirow{2}{*}{\multicolumn{5}{|c|}{$\begin{array}{ll}0.1860 \\
0.0415\end{array}$}} \\
\hline & & & & & & & & & & & & & & & \\
\hline & 0.0005 & 0.0011 & 0.0014 & 0.0022 & 0.0022 & 0.0170 & 0.0377 & 0.0916 & 0.1010 & 0.1017 & 0.0090 & 0.0248 & 0.0271 & 0.0582 & 0.0611 \\
\hline PLST [28] & 0.0017 & 0.0017 & 0.0017 & 0.0017 & 0.0017 & 0.0365 & 0.0503 & 0.0540 & 0.0553 & 0.0557 & 0.0248 & 0.0357 & 0.0397 & 0.0403 & 0.0406 \\
\hline CPLST [30] & 0.0017 & 0.0017 & 0.00 & 0.00 & 0.00 & 0.0443 & 0.0560 & 0.0 & 0.0 & 0.05 & 0.0384 & 0.0400 & 0.04 & 0.0 & 0.0403 \\
\hline MLC-BMaD [31] & 0.0017 & 0.0017 & 0.0017 & 0.0017 & 0.0017 & 0.0341 & 0.0505 & 0.0524 & 0.0550 & 0.0582 & 0.0325 & 0.0354 & 0.0385 & 0.0416 & 0.0415 \\
\hline ML-CSSP [32] & 0.0011 & 0.0016 & 0.0014 & 0.0014 & 0.0015 & 0.0281 & 0.0330 & 0.0439 & 0.0480 & 0.0471 & 0.0184 & 0.0292 & 0.0300 & 0.0302 & 0.0339 \\
\hline $\begin{array}{l}\text { R-E }{ }^{2} \mathrm{FE}(\sim \mathrm{PLST}) \\
\end{array}$ & 0.0017 & 0.0017 & 0.0017 & 0.0017 & 0.0017 & 0.0358 & 0.0498 & 0.0538 & 0.0552 & 0.0556 & 0.0249 & 0.0358 & 0.0398 & 0.0404 & 0.0407 \\
\hline 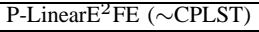 & 0.0017 & 0.0017 & 0.0017 & 0.0017 & 0.0017 & 0.0443 & 0.0548 & 0.0574 & 0.0593 & 0.0601 & 0.0389 & 0.0404 & 0.0417 & 0.0421 & 0.0422 \\
\hline OP-E ${ }^{2} \mathrm{FE}$ & 0.0021 & 0.0021 & 0.0021 & 0.0021 & 0.0024 & 0.0536 & 0.0810 & 0.0906 & 0.0958 & 0.0981 & 0.0400 & 0.0434 & 0.0451 & 0.0454 & 0.0459 \\
\hline LinearE $^{2} \mathrm{FE}$ & 0.0018 & 0.0017 & 0.0017 & 0.0017 & 0.00 & 0.0440 & 0.0564 & 0.0604 & 0.0602 & 0.0601 & 0.0397 & 0.0419 & 0.0423 & 0.0424 & 0.0425 \\
\hline $\mathrm{E}^{2} \mathrm{FE}$ & 0.0026 & 0.0025 & 0.0025 & 0.0025 & 0.0025 & 0.0595 & 0.0888 & 0.1169 & 0.1286 & 0.1369 & 0.0472 & 0.0706 & 0.0752 & 0.0764 & 0.0775 \\
\hline $\begin{array}{l}\text { kernel-CPLST [30] } \\
\end{array}$ & $\overline{0.0019}$ & $\overline{0.0019}$ & 0.0019 & $\overline{0.0019}$ & $\overline{0.0019}$ & 0.0503 & 0.0698 & 0.0728 & 0.0742 & 0.0744 & $\overline{0.0410}$ & 0.0448 & $\overline{0.0462}$ & 0.0472 & 0.0477 \\
\hline kernel- $-\mathrm{E}^{2} \mathrm{FE}$ & 0.0040 & 0.0043 & 0.0043 & 0.0044 & 0.0045 & 0.0629 & 0.0930 & 0.1247 & 0.1396 & 0.1472 & 0.0492 & 0.0699 & 0.0738 & 0.0756 & 0.0770 \\
\hline
\end{tabular}

TABLE V

EXPERIMENTAL RESULTS: example-based Accuracy ON delicious, CAL500, mediamill, ESPGame, bibtex AND bookmarks, WITH VARYING $d_{z} / d_{y}$

\begin{tabular}{|c|c|c|c|c|c|c|c|c|c|c|c|c|c|c|c|}
\hline \multirow{2}{*}{$\frac{\text { Datasets }}{d_{z} / d_{y}}$} & \multicolumn{5}{|c|}{ delicious } & \multicolumn{5}{|c|}{ CAL500 } & \multicolumn{5}{|c|}{ mediamill } \\
\hline & $10 \%$ & $20 \%$ & $30 \%$ & $40 \%$ & $50 \%$ & $10 \%$ & $20 \%$ & $30 \%$ & $40 \%$ & $50 \%$ & $10 \%$ & $20 \%$ & $30 \%$ & $40 \%$ & $50 \%$ \\
\hline \begin{tabular}{l|l} 
& L-SVM \\
\end{tabular} & \multicolumn{5}{|c|}{0.1500} & \multicolumn{5}{|c|}{0.2436} & \multicolumn{5}{|c|}{$\overline{0.3621}$} \\
\hline BR [47] & \multicolumn{5}{|c|}{0.0958} & \multicolumn{5}{|c|}{0.1995} & \multicolumn{5}{|c|}{0.4188} \\
\hline CS [27] & 0.0254 & 0.0540 & 0.0890 & 0.0974 & 0.0964 & 0.1130 & 0.1299 & 0.1626 & 0.1904 & 0.1835 & 0.0115 & 0.0304 & 0.0352 & 0.1425 & 0.1426 \\
\hline PLST [28] & 0.0870 & 0.0898 & 0.0907 & 0.0911 & 0.0912 & 0.2099 & 0.2103 & 0.2103 & 0.2106 & 0.2104 & 0.4160 & 0.4184 & 0.4187 & 0.4188 & 0.4187 \\
\hline CPLST $[30]$ & 0.0954 & 0.0955 & 0.0955 & 0.0955 & 0.0955 & 0.2003 & 0.2007 & 0.2009 & 0.2010 & 0.2010 & 0.4167 & 0.4187 & 0.4187 & 0.4188 & 0.4187 \\
\hline MLC-BMaD [31] & 0.0593 & 0.0700 & 0.0855 & 0.0873 & 0.0875 & 0.1286 & 0.1215 & 0.1194 & 0.1244 & 0.1255 & 0.3989 & 0.3995 & 0.3995 & 0.3995 & 0.3995 \\
\hline ML-CSSP [32] & 0.0684 & 0.0785 & 0.0851 & 0.0893 & 0.0904 & 0.1806 & 0.1880 & 0.1913 & 0.1958 & 0.1966 & 0.3466 & 0.4053 & 0.4073 & 0.4140 & 0.4081 \\
\hline R-E ${ }^{2}$ FE $(\sim$ PLST $)$ & 0.0870 & 0.0898 & 0.0908 & 0.0911 & 0.0913 & 0.2100 & 0.2098 & 0.2099 & 0.2101 & 0.2100 & 0.4159 & 0.4183 & 0.4188 & 0.4188 & 0.4187 \\
\hline P-LinearE ${ }^{2} \mathrm{FE}(\sim \mathrm{CPLST})$ & 0.0984 & 0.1007 & 0.1011 & 0.1011 & 0.1011 & 0.2084 & 0.2189 & 0.2226 & 0.2223 & 0.2223 & 0.4137 & 0.4162 & 0.4182 & 0.4182 & 0.4186 \\
\hline $\mathrm{OP}-\mathrm{E}^{2} \mathrm{FE}$ & 0.1085 & 0.1091 & 0.1093 & 0.1094 & 0.1073 & 0.2283 & 0.2262 & 0.2251 & - & - & 0.4172 & 0.4186 & 0.4189 & 0.4189 & 0.4189 \\
\hline LinearE $^{2} \mathrm{FE}$ & 0.1068 & 0.1055 & 0.1049 & 0.1048 & 0.1048 & 0.2318 & 0.2301 & 0.2291 & 0.2281 & 0.2281 & 0.4182 & 0.4190 & 0.4191 & 0.4190 & 0.4189 \\
\hline $\mathrm{E}^{2} \mathrm{FE}$ & 0.1187 & 0.1196 & 0.1197 & 0.1196 & 0.1184 & 0.2405 & 0.2411 & 0.2421 & 0.2396 & 0.2392 & 0.4353 & 0.4379 & 0.4378 & 0.4378 & 0.4378 \\
\hline "kernel-CPLST [30] & $\overline{0.1116}$ & $\overline{0.1162}$ & $\overline{0.1175}$ & 0.1181 & $\overline{0.1186}$ & $\overline{0.2139}$ & $\overline{0.2148}$ & $\overline{0.2148}$ & 0.2148 & $\overline{0.2148}$ & 0.4489 & $\overline{0.4561}$ & $\overline{0.4572}$ & $\overline{0.4579}$ & $\overline{\overline{0.4580}}$ \\
\hline kernel-E $\mathrm{E}^{2} \mathrm{FE}$ & 0.1281 & 0.1291 & 0.1311 & 0.1312 & 0.1293 & 0.2421 & 0.2414 & 0.2397 & 0.2399 & 0.2398 & 0.4606 & 0.4647 & 0.4681 & 0.4685 & 0.4686 \\
\hline Datasets & \multicolumn{5}{|c|}{ ESPGame } & & & bibtex & & & & & ookmark & & \\
\hline$d_{z} / d_{y}$ & $5 \%$ & $10 \%$ & $15 \%$ & $20 \%$ & $25 \%$ & $10 \%$ & $20 \%$ & $30 \%$ & $40 \%$ & $50 \%$ & $10 \%$ & $20 \%$ & $30 \%$ & $40 \%$ & $50 \%$ \\
\hline Le-SVM & & & 0.0628 & & & & & 0.2827 & & & & & 0.1679 & & \\
\hline DK $[4 /] \stackrel{\text { L-RR }}{ }$ & & & 0.0572 & & & & & 0.1816 & & & & & 0.1597 & & \\
\hline CS [27] & 0.0053 & 0.0057 & 0.0048 & 0.0107 & 0.0106 & 0.0771 & 0.0964 & 0.1435 & 0.1520 & 0.1553 & 0.0155 & 0.0629 & 0.0643 & 0.0940 & 0.0963 \\
\hline PLST [28] & 0.0576 & 0.0576 & 0.0576 & 0.0576 & 0.0576 & 0.1434 & 0.1657 & 0.1760 & 0.1768 & 0.1772 & 0.1472 & 0.1530 & 0.1572 & 0.1575 & 0.1578 \\
\hline CPLST [30] & 0.0578 & 0.0578 & 0.0578 & 0.0578 & 0.0578 & 0.1639 & 0.1768 & 0.1793 & 0.1802 & 0.1800 & 0.1542 & 0.1570 & 0.1572 & 0.1572 & 0.1573 \\
\hline MLC-BMaD [31] & 0.0574 & 0.0573 & 0.0573 & 0.0575 & 0.0573 & 0.1365 & 0.1757 & 0.1767 & 0.1746 & 0.1799 & 0.1494 & 0.1538 & 0.1576 & 0.1597 & 0.1596 \\
\hline ML-CSSP [32] & 0.0409 & 0.0548 & 0.0478 & 0.0541 & 0.0499 & 0.1212 & 0.1205 & 0.1433 & 0.1563 & 0.1573 & 0.1053 & 0.1493 & 0.1249 & 0.1063 & 0.1438 \\
\hline${\mathrm{R}-\mathrm{E}^{2} \mathrm{FE}(\sim \mathrm{PLST})}$ & 0.0575 & 0.0575 & 0.0575 & 0.0575 & 0.0575 & 0.1429 & 0.1653 & 0.1756 & 0.1766 & 0.1769 & 0.1473 & 0.1532 & 0.1574 & 0.1577 & 0.1580 \\
\hline P-LinearE ${ }^{2} \mathrm{FE}(\sim \mathrm{CPLST})$ & 0.0574 & 0.0577 & 0.0578 & 0.0578 & 0.0579 & 0.1598 & 0.1726 & 0.1756 & 0.1783 & 0.1792 & 0.1552 & 0.1574 & 0.1582 & 0.1588 & 0.1590 \\
\hline OP-E ${ }^{2} \mathrm{FE}$ & 0.0622 & 0.0621 & 0.0621 & 0.0621 & 0.0694 & 0.1751 & 0.2041 & 0.2126 & 0.2167 & 0.2181 & 0.1565 & 0.1616 & 0.1623 & 0.1625 & 0.1626 \\
\hline LinearE $^{2} \mathrm{FE}$ & 0.0597 & 0.0593 & 0.0588 & 0.0586 & 0.0584 & 0.1618 & 0.1768 & 0.1820 & 0.1816 & 0.1813 & 0.1559 & 0.1592 & 0.1591 & 0.1590 & 0.1589 \\
\hline $\mathrm{E}^{2} \mathrm{FE}$ & 0.0701 & 0.0701 & 0.0700 & 0.0701 & 0.0701 & $\mathbf{0 . 1 8 3 5}$ & 0.2149 & 0.2356 & 0.2440 & 0.2493 & 0.1659 & 0.1913 & 0.1933 & 0.1937 & 0.1939 \\
\hline "kernel-CPLST [30] & 0.0646 & 0.0645 & 0.0645 & 0.0645 & 0.0645 & 0.1739 & 0.1976 & 0.2005 & 0.2006 & 0.2011 & 0.1588 & 0.1638 & 0.1641 & 0.1643 & $\overline{0.1645}$ \\
\hline kernel-E $\mathrm{E}^{2} \mathrm{FE}$ & 0.0832 & 0.0834 & 0.0834 & 0.0834 & 0.0834 & 0.1910 & 0.2232 & 0.2485 & 0.2596 & 0.2640 & 0.1685 & 0.1937 & 0.1952 & 0.1958 & 0.1961 \\
\hline
\end{tabular}

CPLST, which validates the reasonableness of assuming the columns of the code matrix to be orthonormal. 7) kernel-
$\mathrm{E}^{2} \mathrm{FE}$ outperforms $\mathrm{E}^{2} \mathrm{FE}$ on nearly all datasets, showing its effectiveness to handle the non-linear correlations between 
TABLE VI

Performance Comparisons between LinearE ${ }^{2} \mathrm{FE}, \mathrm{E}^{2} \mathrm{FE}$, ernel- $\mathrm{E}^{2} \mathrm{FE}$ and $\pi$ LinearE $^{2} \mathrm{FE}, \pi \mathrm{E}^{2} \mathrm{FE}$, kernel- $\pi \mathrm{E}^{2} \mathrm{FE}$ on delicious, CAL500, mediamill, ESPGame, bibtex AND bookmarks WITH VARYING $d_{z} / d_{y}$, IN TERMS OF label-based macroFI

\begin{tabular}{|c|c|c|c|c|c|c|c|c|c|c|c|c|c|c|c|}
\hline Datasets & \multicolumn{5}{|c|}{ delicious } & \multicolumn{5}{|c|}{ CAL500 } & \multicolumn{5}{|c|}{ mediamill } \\
\hline$d_{z} / d_{y}$ & $10 \%$ & $20 \%$ & $30 \%$ & $40 \%$ & $50 \%$ & $10 \%$ & $20 \%$ & $30 \%$ & $40 \%$ & $50 \%$ & $10 \%$ & $20 \%$ & $30 \%$ & $40 \%$ & $50 \%$ \\
\hline LinearE $^{2} \mathrm{FE}$ & $\overline{0.0413}$ & $\overline{0.0417}$ & $\overline{\overline{0.0416}}$ & $\overline{0.0416}$ & $\overline{0.0416}$ & 0.1061 & 0.1115 & $\overline{0.1110}$ & 0.1101 & $\overline{0.1101}$ & $\overline{0.0440}$ & $\overline{0.0449}$ & $\overline{0.0451}$ & $\overline{0.0449}$ & $\overline{0.0448}$ \\
\hline$\pi$ LinearE $^{2} \mathrm{FE}$ & 0.0590 & 0.0595 & 0.0594 & 0.0593 & 0.0593 & 0.1415 & 0.1457 & 0.1479 & 0.1477 & 0.1478 & 0.0580 & 0.0605 & 0.0607 & 0.0604 & 0.0603 \\
\hline Relative Improvement & $43.0 \%$ & $42.7 \%$ & $42.5 \%$ & $42.6 \%$ & $42.6 \%$ & $33.3 \%$ & $30.8 \%$ & $33.2 \%$ & $34.1 \%$ & $34.1 \%$ & $31.8 \%$ & $34.7 \%$ & $34.7 \%$ & $34.7 \%$ & $34.7 \%$ \\
\hline $\begin{array}{ll}\mathrm{E}^{2} \mathrm{FE} \\
\end{array}$ & $\overline{0.0530}$ & $\overline{\overline{0.0569}}$ & $\overline{0.0577}$ & 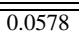 & 0.0578 & $\overline{0.1198}$ & 0.1247 & 0.1263 & 0.1258 & $\overline{0.1256}$ & $\overline{0.0549}$ & 0.0575 & $\overline{0.0577}$ & 0.0577 & $\overline{0.0577}$ \\
\hline$\pi \mathrm{E}^{2} \mathrm{FE}$ & 0.0698 & 0.0727 & 0.0735 & 0.0738 & 0.0717 & 0.1841 & 0.1874 & 0.1883 & 0.1925 & 0.1923 & 0.0685 & 0.0718 & 0.0720 & 0.0721 & 0.0721 \\
\hline Relative Improvement & $31.8 \%$ & $27.8 \%$ & $27.5 \%$ & $27.8 \%$ & $24.0 \%$ & $53.7 \%$ & $50.3 \%$ & $49.0 \%$ & $53.0 \%$ & $53.1 \%$ & $24.9 \%$ & $24.8 \%$ & $24.8 \%$ & $24.9 \%$ & $24.8 \%$ \\
\hline kernel- $-\mathrm{E}^{2} \mathrm{FE}$ & $\overline{\overline{0.0500}}$ & $\overline{\overline{0.0569}}$ & $\overline{\overline{0.0591}}$ & $\overline{\overline{0.0599}}$ & 0.0599 & $\overline{0.1160}$ & 0.1208 & $\overline{0.1215}$ & $\overline{0.1272}$ & 0.1307 & $\overline{\overline{0.0692}}$ & $\overline{\overline{0.0814}}$ & $\overline{\overline{0.0945}}$ & $\overline{\overline{0.0997}}$ & $\overline{0.1003}$ \\
\hline kernel- $\pi \mathrm{E}^{2} \mathrm{FE}$ & 0.0768 & $\overline{0.0820}$ & 0.0834 & 0.0838 & 0.0843 & 0.1827 & 0.1864 & 0.1927 & 0.2000 & 0.2024 & 0.0838 & 0.0975 & 0.1117 & 0.1172 & 0.1177 \\
\hline Relative Improvement & $53.4 \%$ & $44.0 \%$ & $41.1 \%$ & $39.8 \%$ & $40.6 \%$ & $57.4 \%$ & $54.3 \%$ & $58.6 \%$ & $57.3 \%$ & $54.9 \%$ & $21.1 \%$ & $19.8 \%$ & $18.2 \%$ & $17.5 \%$ & $17.3 \%$ \\
\hline
\end{tabular}

\begin{tabular}{|c|c|c|c|c|c|c|c|c|c|c|c|c|c|c|c|}
\hline Datasets & \multicolumn{5}{|c|}{ ESPGame } & \multicolumn{5}{|c|}{ bibtex } & \multicolumn{5}{|c|}{ bookmarks } \\
\hline$d_{z} / d_{y}$ & $5 \%$ & $10 \%$ & $15 \%$ & $20 \%$ & $25 \%$ & $10 \%$ & $20 \%$ & $30 \%$ & $40 \%$ & $50 \%$ & $10 \%$ & $20 \%$ & $30 \%$ & $40 \%$ & $50 \%$ \\
\hline LinearE ${ }^{2} \mathrm{FE}$ & 0.0018 & 0.0017 & 0.0017 & 0.0017 & 0.0017 & 0.0440 & 0.0564 & 0.0604 & 0.0602 & 0.0601 & 0.0397 & 0.0419 & 0.0423 & 0.0424 & 0.0425 \\
\hline$\pi$ LinearE ${ }^{2} \mathrm{FE}$ & 0.0029 & 0.0028 & 0.0028 & 0.0028 & 0.0028 & 0.0801 & 0.1076 & 0.1186 & 0.1217 & 0.1216 & 0.0518 & 0.0569 & 0.0582 & 0.0593 & 0.0592 \\
\hline Relative Improvement & $64.4 \%$ & $63.5 \%$ & $64.0 \%$ & $64.0 \%$ & $64.0 \%$ & $81.9 \%$ & $90.9 \%$ & $96.4 \%$ & $102.2 \%$ & $102.3 \%$ & $30.4 \%$ & $35.8 \%$ & $37.6 \%$ & $39.9 \%$ & $39.4 \%$ \\
\hline $\mathrm{E}^{2} \mathrm{FE}$ & 0.0026 & 0.0025 & 0.0025 & 0.0025 & 0.0025 & 0.0595 & $\overline{0.0888}$ & $\overline{0.1169}$ & 0.1286 & 0.1369 & 0.0472 & 0.0706 & $\overline{0.0752}$ & $\overline{0.0764}$ & $\overline{0.0775}$ \\
\hline$\pi \mathrm{E}^{2} \mathrm{FE}$ & 0.0037 & 0.0037 & 0.0038 & 0.0038 & 0.0038 & 0.1204 & 0.1874 & 0.2264 & 0.2458 & 0.2583 & 0.0751 & 0.0883 & 0.0948 & 0.0976 & 0.0989 \\
\hline Relative Improvement & $43.5 \%$ & $46.5 \%$ & $48.3 \%$ & $48.2 \%$ & $48.0 \%$ & $102.1 \%$ & $111.0 \%$ & $93.7 \%$ & $91.2 \%$ & $88.7 \%$ & $59.1 \%$ & $25.1 \%$ & $26.0 \%$ & $27.7 \%$ & $27.6 \%$ \\
\hline kernel-E $\mathrm{E}^{2} \mathrm{FE}$ & $\overline{0.0040}$ & 0.0043 & 0.0043 & 0.0044 & 0.0045 & 0.0629 & 0.0930 & 0.1247 & 0.1396 & $\overline{0.1472}$ & $\overline{0.0492}$ & 0.0699 & 0.0738 & 0.0756 & 0.0770 \\
\hline kernel- $\pi \mathrm{E}^{2} \mathrm{FE}$ & $\overline{0.0054}$ & 0.0057 & 0.0058 & 0.0059 & 0.0060 & 0.1252 & 0.1936 & 0.2346 & 0.2575 & 0.2740 & 0.0764 & 0.0876 & 0.0936 & 0.0974 & 0.0990 \\
\hline Relative Improvement & $36.4 \%$ & $33.8 \%$ & $34.4 \%$ & $33.6 \%$ & $33.6 \%$ & $99.0 \%$ & $108.2 \%$ & $88.2 \%$ & $84.5 \%$ & $86.1 \%$ & $55.4 \%$ & $25.5 \%$ & $26.9 \%$ & $28.7 \%$ & $28.6 \%$ \\
\hline
\end{tabular}

TABLE VII

Performance Comparisons between LinearE ${ }^{2} \mathrm{FE}, \mathrm{E}^{2} \mathrm{FE}$, kernel- $\mathrm{E}^{2} \mathrm{FE}$ and $\pi$ LinearE $^{2} \mathrm{FE}, \pi \mathrm{E}^{2} \mathrm{FE}$, kernel- $\pi \mathrm{E}^{2} \mathrm{FE}$ on delicious, CAL500, mediamill, ESPGame, bibtex AND bookmarks WITH VARYING $d_{z} / d_{y}$, IN TERMS OF example-based Accuracy

\begin{tabular}{|c|c|c|c|c|c|c|c|c|c|c|c|c|c|c|c|}
\hline Datasets & \multicolumn{5}{|c|}{ delicious } & \multicolumn{5}{|c|}{ CAL500 } & \multicolumn{5}{|c|}{ mediamill } \\
\hline$d_{z} / d_{y}$ & $10 \%$ & $20 \%$ & $30 \%$ & $40 \%$ & $50 \%$ & $10 \%$ & $20 \%$ & $30 \%$ & $40 \%$ & $50 \%$ & $10 \%$ & $20 \%$ & $30 \%$ & $40 \%$ & $50 \%$ \\
\hline "LinearE ${ }^{2} \mathrm{FE}$ & 0.1068 & 0.1055 & 0.1049 & "0.1048 & 0.1048 & 0.2318 & 0.2301 & 0.2291 & 0.2281 & 0.2281 & 0.4182 & 0.4190 & 0.4191 & $\overline{0.4190}$ & $\overline{0.4189}$ \\
\hline & & & & & & & & & & & & & & & 0.4274 \\
\hline Relative Improvement & $37.2 \%$ & $38.0 \%$ & $38.6 \%$ & $38.6 \%$ & $38.6 \%$ & $10.2 \%$ & $8.4 \%$ & $9.1 \%$ & $9.5 \%$ & $9.5 \%$ & $1.8 \%$ & $2.1 \%$ & $2.0 \%$ & $2.0 \%$ & $2.0 \%$ \\
\hline $\mathrm{E}^{2} \mathrm{FE}$ & "0.1187 & $\overline{0.1196}$ & "0.1197 & "0.1196 & $\overline{0.1184}$ & $\overline{0.2405}$ & 0.2411 & $\overline{0.2421}$ & 0.2396 & $\overline{0.2392}$ & $\overline{\overline{0.4353}}$ & $\overline{0.4379}$ & $\overline{0.4378}$ & $\overline{0.4378}$ & $\overline{\mathbf{0 . 4 3 7 8}}$ \\
\hline & 971 & & 0.19 & 4 & 0.1980 & & 0.30 & & 0.3042 & 0.3040 & 0.4280 & 03 & 0.4304 & 0.4305 & 0.4305 \\
\hline Relative Improvement & $66.0 \%$ & $65.1 \%$ & $64.9 \%$ & $65.0 \%$ & $67.2 \%$ & $29.8 \%$ & $26.2 \%$ & $25.5 \%$ & $27.0 \%$ & $27.1 \%$ & $-1.7 \%$ & $-1.7 \%$ & $-1.7 \%$ & $-1.6 \%$ & $-1.7 \%$ \\
\hline kernel-E $\mathrm{E}^{2} \mathrm{FE}$ & 0.1281 & 1291 & $\overline{0.1311}$ & 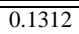 & $\overline{0.1293}$ & $\overline{0.2421}$ & $\overline{0.2414}$ & 0.2397 & 0.2399 & $\overline{0.2398}$ & $\overline{0.4606}$ & $\overline{0.4647}$ & $\overline{0.4681}$ & $\overline{0.4685}$ & $\overline{0.4686}$ \\
\hline & & & & & & & & & & & & 0.4 & 0.4629 & 0.4633 & 0.4632 \\
\hline Relative Improvement & $74.1 \%$ & $73.8 \%$ & $71.3 \%$ & $71.2 \%$ & $73.7 \%$ & $33.1 \%$ & $32.0 \%$ & $32.1 \%$ & $29.3 \%$ & $27.2 \%$ & $-1.6 \%$ & $-0.9 \%$ & $-1.1 \%$ & $-1.1 \%$ & $-1.1 \%$ \\
\hline
\end{tabular}

\begin{tabular}{|c|c|c|c|c|c|c|c|c|c|c|c|c|c|c|c|}
\hline Datasets & \multicolumn{5}{|c|}{ ESPGame } & \multicolumn{5}{|c|}{ bibtex } & \multicolumn{5}{|c|}{ bookmarks } \\
\hline$d_{z} / d_{y}$ & $5 \%$ & $10 \%$ & $15 \%$ & $20 \%$ & $25 \%$ & $10 \%$ & $20 \%$ & $30 \%$ & $40 \%$ & $50 \%$ & $10 \%$ & $20 \%$ & $30 \%$ & $40 \%$ & $50 \%$ \\
\hline 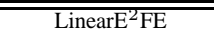 & 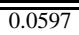 & 0.0593 & 0.0588 & $\overline{0.0586}$ & $\overline{\overline{0.0584}}$ & 0.1618 & 0.1768 & 0.1820 & "0.1816 & 0.1813 & 0.1559 & 0.1592 & $\overline{0.1591}$ & 0.1590 & 0.1589 \\
\hline$\pi$ LinearE $^{2} \mathrm{FE}$ & 0.1024 & 0.1018 & 0.1015 & 0.1013 & 0.1012 & 0.2000 & 0.2276 & 0.2358 & 0.2399 & 0.2407 & 0.1756 & 0.1833 & 0.1835 & 0.1835 & 0.1832 \\
\hline Relative Improvement & $71.4 \%$ & $71.7 \%$ & $72.5 \%$ & $72.8 \%$ & $73.2 \%$ & $23.6 \%$ & $28.7 \%$ & $29.5 \%$ & $32.1 \%$ & $32.8 \%$ & $12.6 \%$ & $15.1 \%$ & $15.3 \%$ & $15.4 \%$ & $15.3 \%$ \\
\hline $\mathrm{E}^{2} \mathrm{FE}$ & 0.0701 & $\overline{0.0701}$ & $\overline{\overline{0.0700}}$ & $\overline{0.0701}$ & $\overline{0.0701}$ & 0.1835 & $\overline{0.2149}$ & 0.2356 & $\overline{0.2440}$ & 0.2493 & 0.1659 & 0.1913 & $\overline{0.1933}$ & 0.1937 & $\overline{\overline{0.1939}}$ \\
\hline$\pi \mathrm{E}^{2} \mathrm{FE}$ & 0.1223 & 0.1303 & 0.1303 & 0.1303 & 0.1302 & 0.2449 & 0.2973 & 0.3219 & 0.3296 & 0.3333 & 0.2089 & 0.2256 & 0.2285 & 0.2294 & 0.2295 \\
\hline Relative Improvement & $74.4 \%$ & $86.0 \%$ & $86.0 \%$ & $85.9 \%$ & $85.8 \%$ & $33.4 \%$ & $38.4 \%$ & $36.6 \%$ & $35.1 \%$ & $33.7 \%$ & $26.0 \%$ & $18.0 \%$ & $18.2 \%$ & $18.4 \%$ & $18.3 \%$ \\
\hline 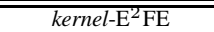 & $\overline{0.0832}$ & 0.0834 & $\overline{0.0834}$ & $\overline{0.0834}$ & $\overline{0.0834}$ & 0.1910 & 0.2232 & $\overline{0.2485}$ & $\overline{0.2596}$ & 0.2640 & 0.1685 & $\overline{\overline{0.1937}}$ & $\overline{\overline{0.1952}}$ & $\overline{\overline{0.1958}}$ & $\overline{\overline{0.1961}}$ \\
\hline kernel- $\pi \mathrm{E}^{2} \mathrm{FE}$ & 0.1334 & 0.1335 & 0.1336 & 0.1336 & 0.1336 & 0.2490 & 0.3045 & 0.3296 & 0.3398 & 0.3463 & 0.2138 & 0.2273 & 0.2296 & 0.2308 & 0.2311 \\
\hline Relative Improvement & $60.4 \%$ & $60.1 \%$ & $60.3 \%$ & $60.2 \%$ & $60.2 \%$ & $30.4 \%$ & $36.4 \%$ & $32.6 \%$ & $30.9 \%$ & $31.2 \%$ & $26.9 \%$ & $17.3 \%$ & $17.6 \%$ & $17.9 \%$ & $17.9 \%$ \\
\hline
\end{tabular}

the feature space and the latent space. Moreover, kernel$\mathrm{E}^{2} \mathrm{FE}$ achieves superior performance to kernel-CPLST, which outperforms CPLST. 8) On all datasets, as $d_{z} / d_{y}$ increases, the performance of $\mathrm{E}^{2} \mathrm{FE}$ does not vary dramatically due to the orthonormality constraint in formula (4), which leads $\mathrm{E}^{2} \mathrm{FE}$ to compactly encode the label space with a smaller $d_{z}$. Similar phenomenon occurs when applying PLST and CPLST, because both are also orthogonally constrained. Actually, we find that this phenomenon still remains when $d_{z} / d_{y}>50 \%$.

Actually, to evaluate the significance of the performance improvements gained by $\mathrm{E}^{2} \mathrm{FE}$ over the baselines, we also perform paired-sample t-test [50] for both label-based macroF1 and example-based Accuracy on all datasets with varying $d_{z} / d_{y}$. Experimental results show that nearly all $P$-values in significance tests are less than the typical significance level 0.01 , and thus the performance improvements gained by $\mathrm{E}^{2} \mathrm{FE}$ over baselines are statistically significant. For details about the experiments of significance tests, one can refer to the supplementary material.

2) Experimental Validation for Enhancing Decoding Matrix with Priori Knowledge: Here on each dataset we compare LinearE ${ }^{2} \mathrm{FE}, \mathrm{E}^{2} \mathrm{FE}$, kernel- $\mathrm{E}^{2} \mathrm{FE}$ with their counterparts that learn the linear decoding matrix with priori knowledge, i.e. $\pi$ LinearE ${ }^{2} \mathrm{FE}, \pi \mathrm{E}^{2} \mathrm{FE}$ and kernel- $\pi \mathrm{E}^{2} \mathrm{FE}$, as presented in Table VI and VII. Note that in all pairwise comparisons, we also present the relative performance improvements gained by 
TABLE VIII

AVERAGE TRAINING COSTS (IN SECONDS) OF COMPARED ALGORITHMS (“PERFORMING LSDR + TRAINING PREDICTIVE MODELS”) WITH $d_{z} / d_{y}=10 \%$.

\begin{tabular}{l|lll|ll|lc}
\multicolumn{2}{l|}{} & \multicolumn{2}{|c|}{ delicious } & \multicolumn{2}{c|}{ ESPGame } & bookmarks \\
\hline \hline \multirow{2}{*}{ BR [47] } & L-SVM & 187.400 & $(0.000+187.400)$ & $20,487.185$ & $(0.000+20,487.185)$ & $24,809.120$ & $(0.000+24,809.120)$ \\
\cline { 2 - 8 } & L-RR & 15.428 & $(0.000+15.428)$ & 54.666 & $(0.000+54.666)$ & 13.310 & $(0.000+13.310)$ \\
\hline \hline CS [27] & $\mathbf{1 . 7 8 2}$ & $(\mathbf{0 . 1 5 0 + 1 . 6 3 2})$ & $\mathbf{7 . 2 3 5}$ & $(\mathbf{1 . 2 2 9}+\mathbf{6 . 0 0 6})$ & $\mathbf{2 . 3 1 6}$ & $(\mathbf{0 . 1 6 9 + 2 . 1 4 7 )}$ \\
\hline PLST [28] & 2.271 & $(0.637+1.635)$ & 11.341 & $(5.310+6.031)$ & 2.360 & $(0.190+2.170)$ \\
\hline CPLST [30] & 2.799 & $(1.033+1.766)$ & 11.594 & $(5.563+6.031)$ & 3.570 & $(1.426+2.143)$ \\
\hline MLC-BMaD [31] & 68.625 & $(66.937+1.688)$ & 585.494 & $(579.388+6.106)$ & 14.475 & $(12.344+2.131)$ \\
\hline ML-CSSP [32] & 2.574 & $(0.780+1.794)$ & 11.074 & $(5.061+6.013)$ & 2.328 & $(0.188+2.140)$ \\
\hline E $^{2} \mathrm{FE}$ & 3.787 & $(1.984+1.803)$ & 16.936 & $(10.877+6.059)$ & 7.039 & $(4.877+2.162)$ \\
\hline
\end{tabular}

TABLE IX

TIME COMPLEXITY OF COMPARED ALGORITHMS TO PERFORM LSDR

\begin{tabular}{l|l} 
& Time Complexity \\
\hline \hline CS [27] & $\mathcal{O}\left(n d_{y} d_{z}\right)$ \\
\hline PLST [28] & $\mathcal{O}\left(n d_{y} d_{z}\right)$ \\
\hline CPLST [30] & $\mathcal{O}\left(\min \left\{n^{2} d_{x}, n d_{x}^{2}\right\}\right)+\mathcal{O}\left(2 n d_{x} d_{y}+d_{x} d_{y}^{2}\right)+\mathcal{O}\left(d_{y}^{3}\right)$ \\
\hline MLC-BMaD [31] & $\mathcal{O}\left(n d_{y}^{2} d_{z}\right)$ \\
\hline ML-CSSP [32] & $\mathcal{O}\left(n d_{y} d_{z}\right)+\mathcal{O}\left(d_{z} \log d_{z}\right)$ \\
\hline \multirow{2}{*}{ E $^{2}$ FE } & $\min \left\{\mathcal{O}\left(n d_{x}^{2}\right)+\mathcal{O}\left(n\left(d_{x}+d_{y}\right)^{2}\right)+\mathcal{O}\left(d_{x} d_{z}^{2}+d_{y} d_{z}^{2}\right)\right.$, \\
& $\left.\mathcal{O}\left(\min \left\{n^{2} d_{x}, n d_{x}^{2}\right\}\right)+\mathcal{O}\left(n^{2} d_{x}+n^{2} d_{y}\right)+\mathcal{O}\left(n d_{z}^{2}\right)\right\}$ \\
\hline
\end{tabular}

\section{$\pi$ LinearE ${ }^{2} \mathrm{FE} / \pi \mathrm{E}^{2} \mathrm{FE} /$ kernel- $\pi \mathrm{E}^{2} \mathrm{FE}$.}

From the comparisons, we can see that in nearly all cases, $\pi$ LinearE $^{2} \mathrm{FE}$ outperforms LinearE ${ }^{2} \mathrm{FE}, \pi \mathrm{E}^{2} \mathrm{FE}$ outperforms $\mathrm{E}^{2} \mathrm{FE}$, and kernel- $\pi \mathrm{E}^{2} \mathrm{FE}$ outperforms kernel- $\mathrm{E}^{2} \mathrm{FE}$. Specifically, on average, considering priori knowledge for learning the decoding matrix can achieve a relative improvement of $48.1 \%$ for label-based macroFl and $33.9 \%$ for examplebased Accuracy. Meanwhile, the maximal gained relative improvement for the former is $111.0 \%$, and that for the latter is $86.0 \%$. Such significant performance improvements well demonstrate the effectiveness of our proposal to further consider the eigenvalues corresponding to each column of the code matrix as priori knowledge to enhance the linear decoding matrix in $\mathrm{E}^{2} \mathrm{FE}$ and its variants.

\section{Analyses of Training Costs}

1) Comparison with Baselines: For $\mathrm{E}^{2} \mathrm{FE}$ and compared baselines, apart from classification performance, here we also compare their training costs theoretically and experimentally.

Considering that the training costs of all algorithms mainly differ in those of performing LSDR, i.e. learning the code vectors of training instances and the decoding process, here we summarize the time complexity of each algorithm, as presented in Table IX. From the time complexity analysis, it can be seen that MLC-BMaD has the highest time complexity, while CS, PLST and ML-CSSP have the lowest.

Moreover, in Table VIII we also report the average time costs for $\mathrm{E}^{2} \mathrm{FE}$ and the compared baselines on performing LSDR and training predictive models over 5 runs on delicious, ESPGame and bookmarks, which have the largest label sets, with $d_{z} / d_{y}=10 \%$. As a reference, the time costs of BR are also provided. All algorithms are conducted with Matlab R2013a on a server with two Intel Xeon E5-2430 CPUs and 64G RAM, except that BR with L-SVM is conducted using LIBLINEAR [48]. Looking at the results of this comparison,
TABLE $X$

AVERAGE TIME COSTS (IN SECONDS) FOR THE NEWLY PROPOSED OPTIMIZATION METHOD ( $\left.E^{2} \mathrm{FE}\right)$ AND THAT PRESENTED IN OUR PREVIOUS CONFERENCE PAPER (FAIE) TO PERFORM LSDR WITH $d_{z} / d_{y}=10 \%$.

\begin{tabular}{l|l|l|l} 
& delicious $^{\circledR}$ & ESPGame $^{\circledR}$ & bookmarks $^{\circledR}$ \\
\hline \hline $\mathrm{E}^{2} \mathrm{FE}$ & 1.077 & 2.646 & 0.683 \\
\hline FaIE [33] & 3.869 & 7.800 & 2.153 \\
\hline
\end{tabular}

we can draw the following conclusions. 1) Compared with BR, nearly all LSDR methods can help to reduce the total training costs. 2) For performing LSDR, $E^{2} \mathrm{FE}$ generally needs slightly higher costs than CS, PLST, CPLST and ML-CSSP, though with superior classification performance. Also, its training cost is much lower than MLC-BMaD. 3) Like the previous theoretical analysis, the training cost of MLC-BMaD is the highest while those of CS, PLST and ML-CSSP are the lowest.

2) Evaluation of the Newly Proposed Optimization Method: To evaluate the optimization method proposed in this paper for efficiently learning the code matrix in cases with $n \gg d_{x}+d_{y}$, we also conduct experiments on delicious, ESPGame and bookmarks to compare its efficiency with that of the optimization method presented in our conference paper [33]. Here we respectively denote the former as $\mathrm{E}^{2} \mathrm{FE}$ and the latter as FaIE.

Considering that FaIE needs to calculate the matrix $\mathbf{U} \in$ $\mathbb{R}^{n \times n}$ and thus needs much memory space for large datasets, to avoid biases brought by high memory space costs, here we follow the experimental settings in [33] and sample 5, 000 training instances for evaluating the time costs of both optimization methods to perform LSDR. Note that here $n \gg d_{x}+d_{y}$ is still ensured for the sampled training instances. Experimental results on the three datasets with $d_{z} / d_{y}=10 \%$ are reported in Table X. It can be seen that the time costs of the newly proposed optimization method are significantly lower than those of the one presented in [33]. That clearly demonstrates the effectiveness of the newly proposed optimization method for cases with $n \gg d_{x}+d_{y}$.

\section{Parameter Sensitivity Analyses}

For a more detailed view, we also conduct experiments to see the effects of $\alpha$ (i.e. formula (12)) on the performance of the proposed $\mathrm{E}^{2} \mathrm{FE}$. Fig. 2 presents how the performance of $\mathrm{E}^{2} \mathrm{FE}$ changes as $\alpha$ varies in $\left\{10^{-2}, 10^{-1}, \cdots, 10^{4}, 10^{5}\right\}$ in a run on the largest delicious, ESPGame and bookmarks with $d_{z} / d_{y}=10 \%$. It can be seen that on these three datasets the performance of $\mathrm{E}^{2} \mathrm{FE}$, in terms of label-based macroFl and 

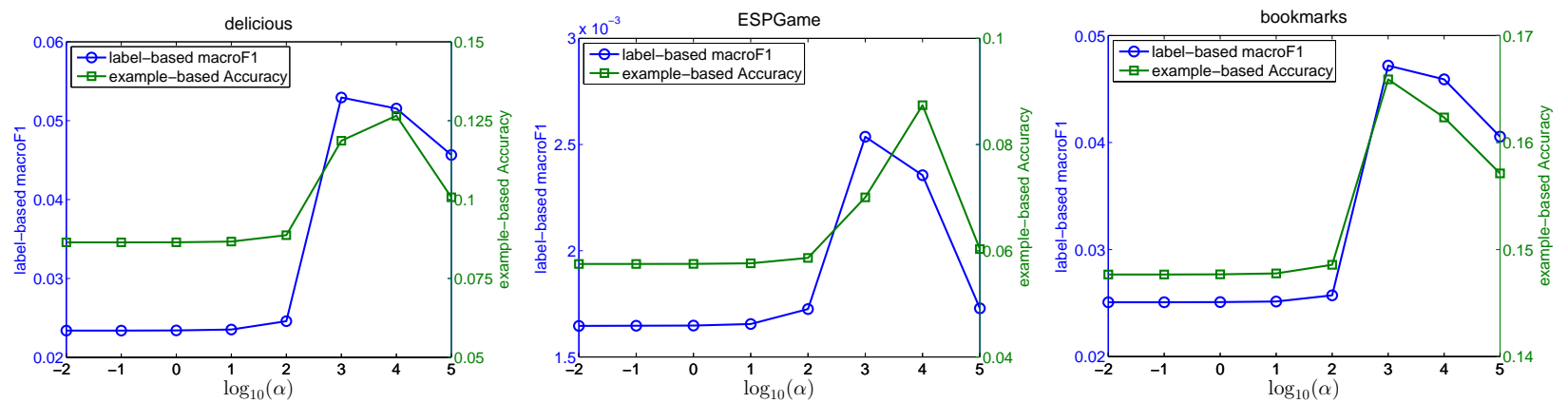

Fig. 2. Effects of $\alpha$ on the performance of $\mathrm{E}^{2} \mathrm{FE}$ on delicious (left), ESPGame (middle) and bookmarks (right), with $d_{z} / d_{y}=10 \%$.
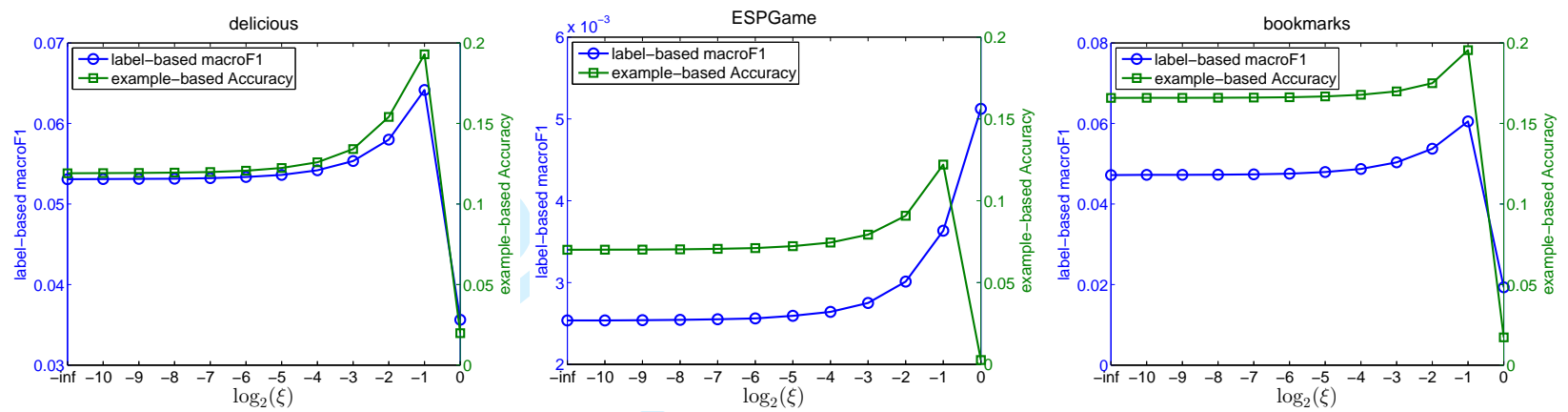

Fig. 3. Effects of $\eta=\xi \frac{1}{\max (\vec{\lambda})}$ on the performance of $\pi \mathrm{E}^{2} \mathrm{FE}$ on delicious (left), ESPGame (middle) and bookmarks (right), with $d_{z} / d_{y}=10 \%$.

example-based Accuracy, firstly increases and then decreases as $\alpha$ increases from $10^{-2}$ to $10^{5}$. That further demonstrates the reasonableness of jointly considering the recoverability of the label space and the predictability of the latent space, as a good trade-off between both yields superior performance. Moreover, we can observe that for these three datasets the optimal $\alpha$ for $\mathrm{E}^{2} \mathrm{FE}$ is near $\left[10^{3}, 10^{4}\right]$.

By fixing $\alpha=10^{3}$, we further analyse the effects of $\eta$ (i.e. formula (18)) on the performance of $\pi \mathrm{E}^{2} \mathrm{FE}$. Specifically, on the largest delicious, ESPGame and bookmarks with $d_{z} / d_{y}=10 \%$, we rewrite $\eta=\xi \frac{1}{\max (\vec{\lambda})}$ and vary $\xi$ in $\left\{0,2^{-10}, 2^{-9}, \ldots, 2^{-1}, 1\right\}$ based on Lemma 3 to see how the corresponding learnt linear decoding matrix affects the performance of $\pi \mathrm{E}^{2} \mathrm{FE}$, as shown in Fig. 3. It can be seen that on the three datasets, as $\eta$ increases from 0 (i.e. $\left.\xi=2^{-i n f}=0\right)$ to $\frac{1}{\max (\vec{\lambda})}\left(\right.$ i.e. $\left.\xi=2^{0}=1\right)$, the performance of $\pi \mathrm{E}^{2} \mathrm{FE}$, in terms of label-based macroF1 and examplebased Accuracy, tends to firstly increase and then decrease in most cases, with the optimal $\eta$ being near $\frac{0.5}{\max (\vec{\lambda})}($ i.e. $\xi=2^{-1}$ ). Actually, $\eta=0$ makes $\pi \mathrm{E}^{2} \mathrm{FE}$ degenerate to $\mathrm{E}^{2} \mathrm{FE}$, and it generally yields inferior performance than $\eta>0$, which further demonstrates the reasonableness of considering the eigenvalues w.r.t columns of the code matrix as priori knowledge to learn an enhanced linear decoding matrix.

\section{DISCUSSIONS}

The proposed $\mathrm{E}^{2} \mathrm{FE}$ assumes that columns of the to-belearnt code matrix $\mathbf{Z}$ are orthonormal. Though this assumption seems to be strong, it is still reasonable and brings useful properties to $E^{2} F E$. 1) As each column of $\mathbf{Z}$ denotes one dimension of the latent space, adding an orthonormality assumption, similar to PLST and CPLST, allows us to mitigate the redundant information among dimensions of the latent space and then enable $\mathrm{E}^{2} \mathrm{FE}$ to encode the label space more compactly. 2) As can be seen in formula (2) - (6), adding the orthonormality assumption can simplify the objective function of $\mathrm{E}^{2} \mathrm{FE}$ and enable it to be transformed into an eigenvalue problem for efficient optimization. 3) By enabling the objective function to be transformed into an eigenvalue problem, from formula (15) and (16) we can see that adding the orthonormality assumption actually helps to ensure $\mathrm{E}^{2} \mathrm{FE}$ obtaining global optima.

Here we also try dropping the orthonormality assumption from the objective function of $\mathrm{E}^{2} \mathrm{FE}$ and conduct experiments on all datasets to evaluate it. We denote it as $\mathrm{E}^{2} \mathrm{FE}_{\text {NoOrth }}$, with its objective function given as follows.

$$
\begin{aligned}
& \Psi=\max _{\mathbf{Z}, \mathbf{Q}}-\|\mathbf{Y}-\mathbf{Z} \mathbf{Q}\|_{\text {fro }}^{2}+\alpha \operatorname{Tr}\left[\mathbf{Z}^{T} \mathbf{H Z}\right] \\
& =\min _{\mathbf{Z}, \mathbf{Q}}\|\mathbf{Y}-\mathbf{Z} \mathbf{Q}\|_{\text {fro }}^{2}-\alpha \operatorname{Tr}\left[\mathbf{Z}^{T} \mathbf{H Z}\right] \\
& \text { s.t. } \quad \forall i \in\left\{1,2, \ldots, d_{z}\right\}, \quad \mathbf{Z}_{\cdot, i}^{T} \mathbf{Z} \mathbf{Z}_{\cdot, i}=1
\end{aligned}
$$

Note that $\mathbf{Z}_{\cdot, i}^{T} \mathbf{Z}_{\cdot, i}=1$ is still required due to formula (7). Like other matrix factorization methods [51], we can derive the code matrix $\mathbf{Z}$ and the linear decoding matrix $\mathbf{Q}$ by using gradient descent methods to iteratively and alternatively optimize one while keeping the other fixed until convergence. For more details, one can refer to the supplementary material.

The performance of $\mathrm{E}^{2} \mathrm{FE}_{\text {NoOrth }}$ on all datasets, using random initial values for $\mathbf{Z}$ and $\mathbf{Q}$ to perform optimization, is presented in Table XI. It can be seen that $\mathrm{E}^{2} \mathrm{FE}_{\text {NoOrth }}$ is inferior to $\mathrm{E}^{2} \mathrm{FE}$. In fact, even using the derived $\mathbf{Z}$ and $\mathrm{Q}$ of $\mathrm{E}^{2} \mathrm{FE}$ as initial values, $\mathrm{E}^{2} \mathrm{FE}_{\text {NoOrth }}$ can hardly gain performance improvement over $\mathrm{E}^{2} \mathrm{FE}$. It is mainly attributed 
TABLE XI

Performance Comparisons BeTWEEN E ${ }^{2} \mathrm{FE}$ AND $\mathrm{E}^{2} \mathrm{FE}_{\text {NoOrth }}$ ON ALL DATASETS, WITH $p=5$ FOR ESPGame AND $p=10$ FOR OTHERS.

\begin{tabular}{|c|c|c|c|c|c|c|c|}
\hline & & $d_{z} / d_{y}$ & $p \%$ & $2 p \%$ & $3 p \%$ & $4 p \%$ & $5 p \%$ \\
\hline \multirow{12}{*}{ 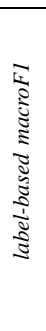 } & \multirow{2}{*}{ delicious } & $\mathrm{E}^{2} \mathrm{FE}$ & 0.0530 & 0.0569 & 0.0577 & 0.0578 & 0.0578 \\
\hline & & $\mathrm{E}^{2} \mathrm{FE}_{\text {NoOrth }}$ & 0.0336 & 0.0401 & 0.0444 & 0.0462 & 0.0438 \\
\hline & \multirow{2}{*}{ CAL500 } & $\mathrm{E}^{2} \mathrm{FE}$ & 0.1198 & 0.1247 & 0.1263 & 0.1258 & 0.1256 \\
\hline & & $\mathrm{E}^{2} \mathrm{FE}_{\text {NoOrth }}$ & 0.0966 & 0.1086 & 0.1074 & 0.1122 & 0.1120 \\
\hline & \multirow{2}{*}{ mediamill } & $\mathrm{E}^{2} \mathrm{FE}$ & 0.0549 & 0.0575 & 0.0577 & 0.0577 & 0.0577 \\
\hline & & $\mathrm{E}^{2} \mathrm{FE}_{\text {NoOrth }}$ & 0.0436 & 0.0448 & 0.0459 & 0.0451 & 0.0453 \\
\hline & \multirow{2}{*}{ ESPGame } & $\mathrm{E}^{2} \mathrm{FE}$ & 0.0026 & 0.0025 & 0.0025 & 0.0025 & 0.0025 \\
\hline & & $\mathrm{E}^{2} \mathrm{FE}_{\text {NoOrth }}$ & 0.0018 & 0.0018 & 0.0017 & 0.0018 & 0.0018 \\
\hline & \multirow{2}{*}{ bibtex } & $\mathrm{E}^{2} \mathrm{FE}$ & 0.0595 & 0.0888 & 0.1169 & 0.1286 & 0.1369 \\
\hline & & $\mathrm{E}^{2} \mathrm{FE}_{\text {NoOrth }}$ & 0.0420 & 0.0727 & 0.0847 & 0.0929 & 0.1000 \\
\hline & \multirow{2}{*}{ bookmarks } & $\mathrm{E}^{2} \mathrm{FE}$ & 0.0472 & 0.0706 & 0.0752 & 0.0764 & 0.0775 \\
\hline & & $\mathrm{E}^{2} \mathrm{FE}_{N o O r t h}$ & 0.0298 & 0.0455 & 0.0459 & 0.0471 & 0.0488 \\
\hline \multirow{12}{*}{ 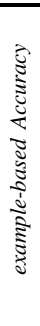 } & \multirow{2}{*}{ delicious } & $\mathrm{E}^{2} \mathrm{FE}$ & 0.1187 & 0.1196 & 0.1197 & 0.1196 & 0.1184 \\
\hline & & $\mathrm{E}^{2} \mathrm{FE}_{N o O r t h}$ & 0.1037 & 0.1073 & 0.1082 & 0.1093 & 0.1045 \\
\hline & \multirow{2}{*}{ CAL500 } & $\mathrm{E}^{2} \mathrm{FE}$ & 0.2405 & 0.2411 & 0.2421 & 0.2396 & 0.2392 \\
\hline & & $\mathrm{E}^{2} \mathrm{FE}_{N o O r t h}$ & 0.2261 & 0.2294 & 0.2256 & 0.2267 & 0.2250 \\
\hline & \multirow{2}{*}{ mediamill } & $\mathrm{E}^{2} \mathrm{FE}$ & 0.4353 & 0.4379 & 0.4378 & 0.4378 & 0.4378 \\
\hline & & $\mathrm{E}^{2} \mathrm{FE}_{N o O r t h}$ & 0.4192 & 0.4201 & 0.4204 & 0.4192 & 0.4199 \\
\hline & \multirow{2}{*}{ ESPGame } & $\mathrm{E}^{2} \mathrm{FE}$ & 0.0701 & 0.0701 & 0.0700 & 0.0701 & 0.0701 \\
\hline & & $\mathrm{E}^{2} \mathrm{FE}_{N o O r t h}$ & 0.0613 & 0.0635 & 0.0605 & 0.0585 & 0.0590 \\
\hline & \multirow{2}{*}{ bibtex } & $\mathrm{E}^{2} \mathrm{FE}$ & 0.1835 & 0.2149 & 0.2356 & 0.2440 & 0.2493 \\
\hline & & $\mathrm{E}^{2} \mathrm{FE}_{\text {NoOrth }}$ & 0.1501 & 0.2018 & 0.2121 & 0.2167 & 0.2233 \\
\hline & \multirow{2}{*}{ bookmarks } & $\mathrm{E}^{2} \mathrm{FE}$ & 0.1659 & 0.1913 & 0.1933 & 0.1937 & 0.1939 \\
\hline & & $\mathrm{E}^{2} \mathrm{FE}_{\text {NoOrth }}$ & 0.1521 & 0.1627 & 0.1636 & 0.1645 & 0.1660 \\
\hline
\end{tabular}

TABLE XII

AVERAGE TIME COSTS (IN SECONDS) FOR PERFORMING LSDR IN $\mathrm{E}^{2} \mathrm{FE}$ AND E ${ }^{2} \mathrm{FE}_{\text {NoOrth }}$ WITH $d_{z} / d_{y}=10 \%$.

\begin{tabular}{l|l|l|l} 
& delicious & ESPGame & bookmarks \\
\hline \hline $\mathrm{E}^{2} \mathrm{FE}$ & 1.984 & 10.877 & 4.877 \\
\hline $\mathrm{E}^{2} \mathrm{FE}_{\text {NoOrth }}$ & 145.253 & 215.199 & 41.434 \\
\hline
\end{tabular}

to that, without the orthonormality assumption, 1) more redundant information rather than complementary information exists between dimensions of the latent space, and 2) global optima cannot be ensured for $\mathbf{Z}$.

Moreover, we also evaluate the time costs for $\mathrm{E}^{2} \mathrm{FE}_{\text {NoOrth }}$ to perform LSDR on the largest delicious, ESPGame and bookmarks with $d_{z} / d_{y}=10 \%$, as reported in Table XII. We can see that $\mathrm{E}^{2} \mathrm{FE}_{\text {NoOrth }}$ costs much more time than $\mathrm{E}^{2} \mathrm{FE}$, because its objective function is more complex for optimization. Its time costs are even higher than those of the binary relevance model L-RR (Table VIII).

Therefore, dropping the orthonormality assumption does not bring substantial performance improvements and instead will increase the time costs for optimization. On the contrary, keeping the orthonormality assumption gains a good trade-off between efficiency and effectiveness for LSDR.

\section{CONCLUSION}

Aiming to address the multi-label classification problem with many classes, in this paper we have proposed an effective method termed $\mathrm{E}^{2} \mathrm{FE}$ to perform LSDR via end-to-end featureaware label space encoding. In contrast to most previous works, $\mathrm{E}^{2} \mathrm{FE}$ requires no encoding functions, and it directly learns a feature-aware code matrix via jointly maximizing the recoverability of the label space and the predictability of the latent space. Subsequently, a linear decoding matrix is further learnt for efficiently recovering the predicted label vectors of unseen instances from their corresponding code vectors generated by trained predictive models. The proposed $\mathrm{E}^{2} \mathrm{FE}$ has close connections to several previous works. It can also be specified to learn an encoding function as previous works, or extended with kernel tricks to handle non-linear correlations between the feature space and the latent space.

\section{REFERENCES}

[1] L. Wang, X. Y. Zhang, and C. Pan, "Msdlsr: Margin scalable discriminative least squares regression for multicategory classification," IEEE Transactions on Neural Networks and Learning Systems, vol. 27, no. 12, pp. 2711-2717, 2016.

[2] J. Ortigosa-Hernndez, I. Inza, and J. A. Lozano, "Semisupervised multiclass classification problems with scarcity of labeled data: A theoretical study," IEEE Transactions on Neural Networks and Learning Systems, vol. 27, no. 12, pp. 2602-2614, 2016.

[3] I. Katakis, G. Tsoumakas, and I. Vlahavas, "Multilabel text classification for automated tag suggestion," in Proceedings of the ECML/PKDD 2008 Discovery Challenge, 2008.

[4] K. Tsoumakas, G. Kalliris, and I. Vlahavas, "Multi-label classification of music into emotions," in Proceedings of the 9th International Conference of Music Information Retrieval, 2008.

[5] A. Kapoor, R. Viswanathan, and P. Jain, "Multilabel classification using bayesian compressed sensing," in Advances in Neural Information Processing Systems, 2012.

[6] B. Taskar, C. Guestrin, and D. Koller, "Max-margin markov networks," Advances in Neural Information Processing Systems, 2003.

[7] M. Boutell, J. Luo, X. Shen, and C. Brown, "Learning multi-label scene classification," Pattern Recognition, vol. 37, no. 9, pp. 1757-1771, 2004.

[8] T. Evgeniou, C. Micchelli, and M. Pontil, "Learning multiple tasks with kernel methods," in Journal of Machine Learning Research, 2005, pp. 615-637.

[9] I. Tsochantaridis, T. Joachims, T. Hofmann, Y. Altun, and Y. Singer, "Large margin methods for structured and interdependent output variables," Journal of Machine Learning Research, vol. 6, no. 2, p. 1453, 2006.

[10] G. Tsoumakas and I. Katakis, "Multi-label classification: An overview," International Journal of Data Warehousing and Mining, vol. 3, no. 3, pp. 1-13, 2007.

[11] A. Argyriou, T. Evgeniou, and M. Pontil, "Convex multi-task feature learning," Machine Learning, vol. 73, no. 3, pp. 243-272, 2008.

[12] B. Hariharan, L. Zelnik-Manor, S. Vishwanathan, and M. Varma, "Large scale max-margin multi-label classification with priors," in Proceedings of the 27th International Conference on Machine Learning, 2010.

[13] G. Tsoumakas, I. Katakis, and I. Vlahavas, "Mining multi-label data," in Data Mining and Knowledge Discovery Handbook. Springer, 2010, pp. 667-685.

[14] T. Zhou and D. Tao, "Multi-label subspace ensemble," in International Conference on Artificial Intelligence and Statistics, 2012.

[15] C. Ferng and H. Lin, "Multilabel classification using error-correcting codes of hard or soft bits," IEEE Transactions on Neural Networks and Learning Systems, vol. 24, no. 11, pp. 1888-1900, 2013.

[16] W. Bi and J. Kwok, "Mandatory leaf node prediction in hierarchical multilabel classification," IEEE Transactions on Neural Networks and Learning Systems, vol. 25, no. 12, pp. 2275-2287, 2014.

[17] Q. Wu, Y. Ye, H. Zhang, T. Chow, and S. Ho, "Ml-tree: A tree-structurebased approach to multilabel learning," IEEE Transactions on Neural Networks and Learning Systems, vol. 26, no. 3, pp. 430-443, 2015.

[18] Y. Luo, D. Tao, C. Xu, C. Xu, H. Liu, and Y. Wen, "Multiview vectorvalued manifold regularization for multilabel image classification," IEEE Transactions on Neural Networks and Learning Systems, vol. 24, no. 5, pp. 709-722, 2013.

[19] S. Chen, X. Yang, and Y. Tian, "Discriminative hierarchical k-means tree for large-scale image classification," IEEE Transactions on Neural Networks and Learning Systems, vol. 26, no. 9, pp. 2200-2205, 2015.

[20] X. Cao, C. Zhang, H. Fu, X. Guo, and Q. Tian, "Saliency-aware nonparametric foreground annotation based on weakly labeled data," IEEE Transactions on Neural Networks and Learning Systems, vol. 27, no. 6, pp. 1253-1265, 2016.

[21] F. Charte, A. Rivera, M. del Jesus, and F. Herrera, "Li-mlc: A label inference methodology for addressing high dimensionality in the label space for multilabel classification," IEEE Transactions on Neural Networks and Learning Systems, vol. 25, no. 10, pp. 1842-1854, 2014. 
[22] D. Bouzas, N. Arvanitopoulos, and A. Tefas, "Graph embedded nonparametric mutual information for supervised dimensionality reduction," IEEE Transactions on Neural Networks and Learning Systems, vol. 26, no. 5 , pp. 951-963, 2015

[23] R. Mall and J. A. K. Suykens, "Very sparse lssvm reductions for large-scale data," IEEE Transactions on Neural Networks and Learning Systems, vol. 26, no. 5, pp. 1086-1097, 2015.

[24] J. Wu and H. Yang, "Linear regression-based efficient svm learning for large-scale classification," IEEE Transactions on Neural Networks and Learning Systems, vol. 26, no. 10, pp. 2357-2369, 2015.

[25] Z. Lai, W. K. Wong, Y. Xu, J. Yang, and D. Zhang, "Approximate orthogonal sparse embedding for dimensionality reduction," IEEE Transactions on Neural Networks and Learning Systems, vol. 27, no. 4, pp. 723-735, 2016.

[26] Y. Yu, K. I. Diamantaras, T. McKelvey, and S. Y. Kung, "Class-specific subspace kernel representations and adaptive margin slack minimization for large scale classification," IEEE Transactions on Neural Networks and Learning Systems, vol. PP, no. 99, pp. 1-17, 2016.

[27] D. Hsu, S. Kakade, J. Langford, and T. Zhang, "Multi-label prediction via compressed sensing," Advances in Neural Information Processing Systems, 2009.

[28] F. Tai and H. Lin, "Multi-label classification with principle label space transformation," in Proceedings of the 2nd International Workshop on Learning from Multi-Label Data, 2010.

[29] T. Zhou, D. Tao, and X. Wu, "Compressed labeling on distilled labelsets for multi-label learning," Machine Learning, vol. 88, no. 1-2, pp. 69126, 2012.

[30] Y. Chen and H. Lin, "Feature-aware label space dimension reduction for multi-label classification," in Advances in Neural Information Processing Systems, 2012.

[31] J. Wicker, B. Pfahringer, and S. Kramer, "Multi-label classification using boolean matrix decomposition," in Proceedings of the 27th Annual ACM Symposium on Applied Computing, 2012.

[32] W. Bi and J. Kwok, "Efficient multi-label classification with many labels," in Proceedings of the 30th International Conference on Machine Learning, 2013.

[33] Z. Lin, G. Ding, M. Hu, and J. Wang, "Multi-label classification via feature-aware implicit label space encoding," in Proceedings of the 31st International Conference on Machine Learning, 2014.

[34] G. Tsoumakas, I. Katakis, and I. Vlahavas, "Effective and efficient multilabel classification in domains with large number of labels," in Proceedings of ECML/PKDD 2008 Workshop on Mining Multidimensional Data, 2008.

[35] O. Dekel and O. Shamir, "Multiclass-multilabel classification with more classes than examples," in International Conference on Artificial Intelligence and Statistics, 2010.

[36] K. Balasubramanian and G. Lebanon, "The landmark selection method for multiple output prediction," in Proceedings of the 29th International Conference on Machine Learning, 2012.

[37] D. Needell and J. Tropp, "Cosamp: Iterative signal recovery from incomplete and inaccurate samples," Applied and Computational Harmonic Analysis, vol. 26, no. 3, pp. 301-321, 2009.

[38] I. Jolliffe, Principal component analysis. Springer-Verlag New York, 1986, vol. 487.

[39] G. Tsoumakas and I. Katakis, "Multi label classification: An overview," International Journal of Data Warehouse and Mining, vol. 3, no. 3, pp. $1-13,2007$

[40] Y. Zhang and J. Schneider, "Multi-label output codes using canonical correlation analysis," in International Conference on Artificial Intelligence and Statistics, 2011.

[41] R. A. Horn and C. R. Johnson, Matrix Analysis. Cambridge University Press, 1990.

[42] R. B. Lehoucq and D. C. Sorensen, "Deflation techniques for an implicitly restarted arnoldi iteration," SIAM Journal on Matrix Analysis and Applications, vol. 17, no. 4, pp. 789-821, 1996.

[43] M. Hu, Y. Chen, and J. Kwok, "Building sparse multiple-kernel svm classifiers," IEEE Transactions on Neural Networks, vol. 20, no. 5, pp. 827-839, 2009.

[44] G. Tsoumakas, E. Spyromitros-Xioufis, J. Vilcek, and I. Vlahavas, "Mulan: A java library for multi-label learning," Journal of Machine Learning Research, vol. 12, pp. 2411-2414, 2011.

[45] L. Von Ahn and L. Dabbish, "Labeling images with a computer game," in Proceedings of the SIGCHI conference on Human factors in computing systems, 2004.

[46] M. Lux and S. A. Chatzichristofis, "Lire: lucene image retrieval: an extensible java cbir library," in Proceedings of the 16th ACM international conference on Multimedia, 2008.
[47] J. Fürnkranz, E. Hüllermeier, E. Loza Mencía, and K. Brinker, "Multilabel classification via calibrated label ranking," Machine Learning, vol. 73, no. 2, pp. 133-153, 2008.

[48] R. Fan, K. Chang, C. Hsieh, X. Wang, and C. Lin, "Liblinear: A library for large linear classification," Journal of Machine Learning Research, vol. 9, pp. 1871-1874, 2008.

[49] M. Zhang and Z. Zhou, "A review on multi-label learning algorithms," IEEE Transactions on Knowledge and Data Engineering, vol. PP, no. 99, pp. 1-1, 2013.

[50] J. McDonald, Handbook of Biological Statistics (3rd ed.). Sparky House Publishing, 2014.

[51] G. Ding, Y. Guo, and J. Zhou, "Collective matrix factorization hashing for multimodal data," in The IEEE Conference on Computer Vision and Pattern Recognition, 2014.

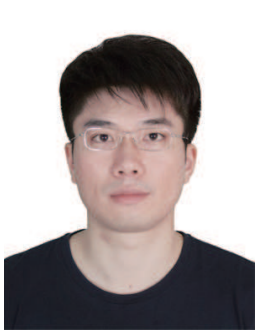

Zijia Lin received his Ph.D. degree from Department of Computer Science and Technology, Tsinghua University, Beijing, China in 2016 and his B.Sc. degree from School of Software in the same campus in 2011. His research interests include multimedia information retrieval and machine learning.

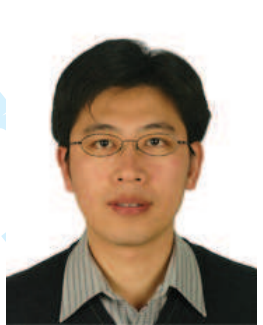

Guiguang Ding received his Ph.D. degree in electronic engineering from the University of Xidian, China. He is currently an associate professor of School of Software, Tsinghua University. Before joining school of software in 2006, he had been a postdoctoral research fellow in the Department of Automation, Tsinghua University. He has published over 70 referred journal and conference papers in TKDE, CVIU, TIST, ICCV, CVPR, ICML, IJCAI, AAAI, etc., and applied for over 20 Patents.

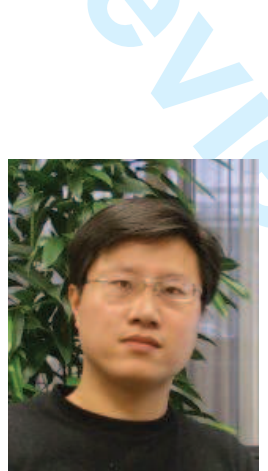

Jungong Han is currently a Senior Lecturer with the Department of Computer Science and Digital Technologies, Northumbria University, Newcastle upon Tyne, U.K. His current research interests include multimedia content identification, multisensor data fusion, computer vision, and multimedia security. Dr. Han is an Associate Editor of Neurocomputing (Elsevier), and an Editorial Board Member of Multimedia Tools and Applications (Springer).

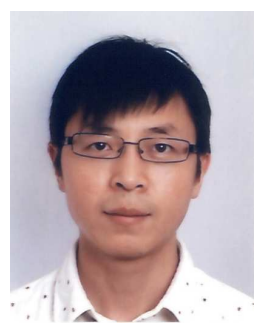

Ling Shao (M09-SM10) is a professor with the School of Computing Sciences at the University of East Anglia, Norwich, UK. Previously, he was a professor (2014-2016) with Northumbria University, a senior lecturer (2009-2014) with the University of Shefeld and a senior scientist (2005-2009) with Philips Research, The Netherlands. His research interests include computer vision, image/video processing and machine learning. $\mathrm{He}$ is an associate editor of IEEE Transactions on Image Processing, IEEE Transactions on Neural Networks and Learning Systems and several other journals. He is a Fellow of the British Computer Society and the Institution of Engineering and Technology. He is a senior member of the IEEE. 


\title{
Supplementary Material for "End-to-End Feature-aware Label Space Encoding for Multi-label Classification with Many Classes"
}

\author{
Zijia Lin, Student Member, IEEE, Guiguang Ding, Member, IEEE, \\ Jungong Han, Ling Shao, Senior Member, IEEE,
}

\section{Proofs For LEMMA 1 AND LEMMA 3}

Lemma 1. For $E^{2} F E$, its RMSE is bounded by

$$
R M S E \leq \frac{2}{\sqrt{n}}\left(\sqrt{d_{z}}\|\mathbf{Y}\|_{\text {fro }}\|\mathbf{Z}-\mathcal{G}(\mathbf{X})\|_{\text {fro }}+\|\mathbf{Y}-\mathbf{Z Q}\|_{\text {fro }}\right)
$$

Proof. RMSE for $\mathrm{E}^{2} \mathrm{FE}$ is defined as follows.

$$
R M S E=\frac{1}{\sqrt{n}}\|\operatorname{round}(\mathcal{G}(\mathbf{X}) \mathbf{Q})-\mathbf{Y}\|_{\text {fro }}
$$

where $\mathcal{G}$ denotes the learnt predictive models for mapping instance features into code vectors, and $\operatorname{round}(\mathcal{G}(\mathbf{X}) \mathbf{Q})$ denotes the recovered binary tagging matrix.

For clarification, we introduce a matrix $\mathbf{R}$ defined as follows.

$$
\mathbf{R}=\operatorname{round}(\mathcal{G}(\mathbf{X}) \mathbf{Q})-\mathbf{Y}
$$

Then for any entry $\mathbf{R}_{i, j}$, we have $\mathbf{R}_{i, j} \in\{-1,0,1\}$ and thus $\mathbf{R}_{i, j}^{2} \in\{0,1\}$.

According to the round $(\cdot)$ operation, there are two cases making $\mathbf{R}_{i, j}^{2}=1$, i.e. 1) $(\mathcal{G}(\mathbf{X}) \mathbf{Q})_{i, j} \geq \frac{1}{2}$ while $\mathbf{Y}_{i, j}=0$, and 2) $(\mathcal{G}(\mathbf{X}) \mathbf{Q})_{i, j}<\frac{1}{2}$ while $\mathbf{Y}_{i, j}=1$. In both cases, we have the following inequality.

$$
\left((\mathcal{G}(\mathbf{X}) \mathbf{Q})_{i, j}-\mathbf{Y}_{i, j}\right)^{2} \geq\left(\frac{1}{2}\right)^{2}=\frac{1}{4} \mathbf{R}_{i, j}^{2}
$$

Actually, when $\mathbf{R}_{i, j}^{2}=0$, the inequality above still holds. Then, we have $\sum_{i, j} \mathbf{R}_{i, j}^{2} \leq 4 \sum_{i, j}\left((\mathcal{G}(\mathbf{X}) \mathbf{Q})_{i, j}-\mathbf{Y}_{i, j}\right)^{2}$. With matrix notations, we can further derive:

$$
\|\mathbf{R}\|_{\text {fro }} \leq 2\|\mathcal{G}(\mathbf{X}) \mathbf{Q}-\mathbf{Y}\|_{\text {fro }}
$$

With the triangle inequality and the Cauchy Schwarz inequality, we can derive that: 1) $\forall \mathbf{A}, \mathbf{B},\|\mathbf{A}+\mathbf{B}\|_{\text {fro }} \leq\|\mathbf{A}\|_{\text {fro }}+$ $\|\mathbf{B}\|_{\text {fro }}$, and 2) $\forall \mathbf{A}, \mathbf{B},\|\mathbf{A B}\|_{\text {fro }} \leq\|\mathbf{A}\|_{\text {fro }}\|\mathbf{B}\|_{\text {fro }}$. Moreover, with $\mathbf{Z}^{T} \mathbf{Z}=\mathbf{I}$, we have $\|\mathbf{Z}\|_{\text {fro }}=\sqrt{d_{z}}$. Then we can utilize them with $\mathbf{Q}=\mathbf{Z}^{T} \mathbf{Y}$ to have the following derivations.

$$
\begin{aligned}
& \|\mathcal{G}(\mathbf{X}) \mathbf{Q}-\mathbf{Y}\|_{\text {fro }} \\
& =\|(\mathcal{G}(\mathbf{X}) \mathbf{Q}-\mathbf{Z Q})+(\mathbf{Z Q}-\mathbf{Y})\|_{\text {fro }} \\
& \leq\|\mathcal{G}(\mathbf{X}) \mathbf{Q}-\mathbf{Z} \mathbf{Q}\|_{\text {fro }}+\|\mathbf{Z} \mathbf{Q}-\mathbf{Y}\|_{\text {fro }} \\
& \leq\|\mathcal{G}(\mathbf{X})-\mathbf{Z}\|_{\text {fro }}\|\mathbf{Q}\|_{\text {fro }}+\|\mathbf{Z} \mathbf{Q}-\mathbf{Y}\|_{\text {fro }} \\
& =\|\mathbf{Z}-\mathcal{G}(\mathbf{X})\|_{\text {fro }}\left\|\mathbf{Z} \mathbf{Z}^{T} \mathbf{Y}\right\|_{\text {fro }}+\|\mathbf{Y}-\mathbf{Z Q}\|_{\text {fro }} \\
& \leq\|\mathbf{Z}-\mathcal{G}(\mathbf{X})\|_{\text {fro }}\|\mathbf{Z}\|_{\text {fro }}\|\mathbf{Y}\|_{\text {fro }}+\|\mathbf{Y}-\mathbf{Z Q}\|_{\text {fro }} \\
& =\sqrt{d_{z}}\|\mathbf{Y}\|_{\text {fro }}\|\mathbf{Z}-\mathcal{G}(\mathbf{X})\|_{\text {fro }}+\|\mathbf{Y}-\mathbf{Z Q}\|_{\text {fro }}
\end{aligned}
$$

Combining formula (1), (2), (4) and (5), we can derive:

$$
R M S E \leq \frac{2}{\sqrt{n}}\left(\sqrt{d_{z}}\|\mathbf{Y}\|_{\text {fro }}\|\mathbf{Z}-\mathcal{G}(\mathbf{X})\|_{\text {fro }}+\|\mathbf{Y}-\mathbf{Z Q}\|_{\text {fro }}\right)
$$

Lemma 3. For any $\eta \in\left[0, \frac{1}{\max (\vec{\lambda})}\right]$ with $\max (\vec{\lambda})$ being the maximal value of $\vec{\lambda}, \tilde{\mathcal{L}}$ will be non-trivial for optimization.

Proof. In $\pi \mathrm{E}^{2} \mathrm{FE}$, the objective function for the enhanced linear decoding matrix $\mathbf{Q}$ is defined as follows.

$$
\tilde{\mathcal{L}}=\min _{\mathbf{Q}}\|\mathbf{Y}-\mathbf{Z Q}\|_{\text {fro }}^{2}-\eta \operatorname{Tr}\left[\mathbf{Q}^{T} \tilde{\mathbf{\Lambda}} \mathbf{Q}\right]
$$

where $\tilde{\Lambda}$ is a diagonal matrix with $\tilde{\Lambda}_{j, j}=\lambda_{j}$, and $\lambda_{j}$ is the eigenvalue corresponding to the $j$ th column of the code matrix Z. Moreover, here $\vec{\lambda}=\left[\lambda_{1}, \lambda_{2}, \ldots, \lambda_{d_{z}}\right]$.

Then $\tilde{\mathcal{L}}$ can be rewritten as the following Quadratic Programming form.

$$
\begin{aligned}
\tilde{\mathcal{L}} & =\min _{\mathbf{Q}} \operatorname{Tr}\left[\mathbf{Q}^{T}\left(\mathbf{Z}^{T} \mathbf{Z}-\eta \tilde{\mathbf{\Lambda}}\right) \mathbf{Q}-2 \mathbf{Y}^{T} \mathbf{Z} \mathbf{Q}\right] \\
& =\min _{\mathbf{Q}} \operatorname{Tr}\left[\mathbf{Q}^{T}(\mathbf{I}-\eta \tilde{\mathbf{\Lambda}}) \mathbf{Q}-2 \mathbf{Y}^{T} \mathbf{Z} \mathbf{Q}\right]
\end{aligned}
$$

where $\mathbf{I}$ is an identity matrix. To make $\tilde{\mathcal{L}}$ non-trivial for optimization, $(\mathbf{I}-\eta \tilde{\boldsymbol{\Lambda}})$ needs to be positive semi-definite. Knowing that $\tilde{\Lambda}$ is a diagonal matrix with its diagonal entries being $\vec{\lambda},(\mathbf{I}-\eta \tilde{\boldsymbol{\Lambda}})$ is also diagonal, and all its diagonal entries are non-negative when $\eta \in\left[0, \frac{1}{\max (\vec{\lambda})}\right]$ with $\max (\vec{\lambda})$ being the maximal value of $\vec{\lambda}$. In that case $(\mathbf{I}-\eta \tilde{\mathbf{\Lambda}})$ is positive semidefinite, making $\tilde{\mathcal{L}}$ non-trivial for optimization.

\section{SupPlementary EXPERIMENTAL RESUlts}

\section{A. Statistical Significance of the Superiority of $E^{2} F E$}

As mentioned in the paper (section VII-B1), to evaluate the significance of the performance improvements gained by $\mathrm{E}^{2} \mathrm{FE}$ over the baselines, we further perform paired-sample t-test [1] for both label-based macroFl and example-based Accuracy on all datasets with varying $d_{z} / d_{y}$.

For label-based macroFl, we take the corresponding $F 1$ values (i.e. $\frac{2 p_{i} r_{i}}{p_{i}+r_{i}}$ in formula (28)) of all labels for each algorithm as the samples from its $F 1$ distribution. Meanwhile, for example-based Accuracy, we take the corresponding accuracy values (i.e. $\frac{\left|G_{j}^{\prime} \cap P_{j}^{\prime}\right|}{\left|G_{j}^{\prime} \cup P_{j}^{\prime}\right|}$ in formula (29)) of all test instances for each algorithm as the samples from its accuracy distribution. Then for each performance metric, we compare the samples of 
TABLE I

RESULTS OF SIGNIFICANCE TEST (i.e. P-value) FOR label-based macroFl BETWEEN E $^{2}$ FE AND THE COMPARED BASELINES ON ALL DATASETS WITH VARYING $d_{z} / d_{y}$

\begin{tabular}{|c|c|c|c|c|c|c|c|c|c|c|c|c|c|c|c|}
\hline Datasets & \multicolumn{5}{|c|}{ delicious } & \multicolumn{5}{|c|}{ CAL500 } & \multicolumn{5}{|c|}{ mediamill } \\
\hline$d_{z} / d_{y}$ & $10 \%$ & $20 \%$ & $30 \%$ & $40 \%$ & $50 \%$ & $10 \%$ & $20 \%$ & $30 \%$ & $40 \%$ & $50 \%$ & $10 \%$ & $20 \%$ & $30 \%$ & $40 \%$ & $50 \%$ \\
\hline CS & $\epsilon$ & $\epsilon$ & $\epsilon$ & $\epsilon$ & $\epsilon$ & $\epsilon$ & $\epsilon$ & $3.57 \mathrm{e}-08$ & $4.19 \mathrm{e}-08$ & $2.25 \mathrm{e}-06$ & $1.73 \mathrm{e}-10$ & $2.11 \mathrm{e}-08$ & $8.27 \mathrm{e}-09$ & $6.87 \mathrm{e}-05$ & $1.76 \mathrm{e}-05$ \\
\hline PLST & $\bar{\epsilon}$ & $\bar{\epsilon}$ & $\bar{\epsilon}$ & $\bar{\epsilon}$ & $\bar{\epsilon}$ & $\bar{\epsilon}$ & $\bar{\epsilon}$ & $\epsilon$ & $\epsilon$ & $\epsilon$ & $\epsilon$ & $\epsilon$ & $\bar{\epsilon}$ & $\epsilon$ & $\epsilon$ \\
\hline $\begin{array}{l}\text { CPLST } \\
\end{array}$ & $\bar{\epsilon}$ & $\bar{\epsilon}$ & $\bar{\epsilon}$ & $\epsilon$ & $\epsilon$ & $\bar{\epsilon}$ & $\bar{\epsilon}$ & $\epsilon$ & $\bar{\epsilon}$ & $\bar{\epsilon}$ & $\bar{\epsilon}$ & $\epsilon$ & $\epsilon$ & $\bar{\epsilon}$ & $\bar{\epsilon}$ \\
\hline MLC-BMaD & $\epsilon$ & $\epsilon$ & $\epsilon$ & $\epsilon$ & $\epsilon$ & $\epsilon$ & $\epsilon$ & $\epsilon$ & $\epsilon$ & $\epsilon$ & $\epsilon$ & $\epsilon$ & $\epsilon$ & $\epsilon$ & $\epsilon$ \\
\hline ML-CSSP & $\bar{\epsilon}$ & $\bar{\epsilon}$ & $\bar{\epsilon}$ & $\bar{\epsilon}$ & $\bar{\epsilon}$ & $\epsilon$ & $\bar{\epsilon}$ & $\bar{\epsilon}$ & $\bar{\epsilon}$ & $\bar{\epsilon}$ & $2.61 \mathrm{e}-09$ & $6.16 \mathrm{e}-10$ & $\bar{\epsilon}$ & $\bar{\epsilon}$ & $\bar{\epsilon}$ \\
\hline Datasets & \multicolumn{5}{|c|}{ ESPGame } & \multicolumn{5}{|c|}{ bibtex } & \multicolumn{5}{|c|}{ bookmarks } \\
\hline$d_{z} / d_{y}$ & $5 \%$ & $10 \%$ & $15 \%$ & $20 \%$ & $25 \%$ & $10 \%$ & $20 \%$ & $30 \%$ & $40 \%$ & $50 \%$ & $10 \%$ & $20 \%$ & $30 \%$ & $40 \%$ & $50 \%$ \\
\hline CS & $\epsilon$ & $3.41 \mathrm{e}-05$ & 0.0015 & 0.206 & 0.181 & $\epsilon$ & $\epsilon$ & $9.1 \mathrm{e}-05$ & $4.16 \mathrm{e}-05$ & $4.65 \mathrm{e}-07$ & $\epsilon$ & $\epsilon$ & $\epsilon$ & 0.000371 & 0.00152 \\
\hline $\begin{array}{l}\text { PLST } \\
\end{array}$ & $\bar{\epsilon}$ & $\epsilon$ & $\epsilon$ & $\epsilon$ & $\epsilon$ & $1.19 \mathrm{e}-10$ & $\epsilon$ & $\epsilon$ & $\epsilon$ & $\epsilon$ & $\epsilon$ & $\epsilon$ & $\epsilon$ & $\epsilon$ & $\epsilon$ \\
\hline CPLST & $\bar{\epsilon}$ & $\bar{\epsilon}$ & $\bar{\epsilon}$ & $\bar{\epsilon}$ & $\bar{\epsilon}$ & $\epsilon$ & $\epsilon$ & $\bar{\epsilon}$ & $\bar{\epsilon}$ & $\bar{\epsilon}$ & $4.3 \mathrm{e}-05$ & $\bar{\epsilon}$ & $\bar{\epsilon}$ & $\bar{\epsilon}$ & $\bar{\epsilon}$ \\
\hline MLC-BMaD & $\bar{\epsilon}$ & $\bar{\epsilon}$ & $\bar{\epsilon}$ & $\bar{\epsilon}$ & $\bar{\epsilon}$ & $1.87 \mathrm{e}-09$ & $\bar{\epsilon}$ & $\bar{\epsilon}$ & $\bar{\epsilon}$ & $\bar{\epsilon}$ & $\epsilon$ & $\epsilon$ & $\epsilon$ & $\epsilon$ & $\bar{\epsilon}$ \\
\hline ML-CSSP & $\epsilon$ & $\epsilon$ & $\epsilon$ & $\epsilon$ & $\epsilon$ & $\epsilon$ & $\epsilon$ & $\epsilon$ & $\epsilon$ & $\epsilon$ & $\epsilon$ & $\epsilon$ & $\epsilon$ & $\epsilon$ & $\epsilon$ \\
\hline
\end{tabular}

TABLE II

RESUlts of SIGNIFICANCE TEST (i.e. P-value) FOR example-based Accuracy BETWEen E ${ }^{2}$ FE AND the COMPARED BASELINES ON ALL DATASETS WITH $\operatorname{VARYING} d_{z} / d_{y}$

\begin{tabular}{|c|c|c|c|c|c|c|c|c|c|c|c|c|c|c|c|}
\hline Datasets & \multicolumn{5}{|c|}{ delicious } & \multicolumn{5}{|c|}{ CAL500 } & \multicolumn{5}{|c|}{ mediamill } \\
\hline$d_{z} / d_{y}$ & $10 \%$ & $20 \%$ & $30 \%$ & $40 \%$ & $50 \%$ & $10 \%$ & $20 \%$ & $30 \%$ & $40 \%$ & $50 \%$ & $10 \%$ & $20 \%$ & $30 \%$ & $40 \%$ & $50 \%$ \\
\hline $\mathrm{CS}$ & $\epsilon$ & $\epsilon$ & $\epsilon$ & $\epsilon$ & $\epsilon$ & $\epsilon$ & $\epsilon$ & $\epsilon$ & $\epsilon$ & $\epsilon$ & $\epsilon$ & $\epsilon$ & $\epsilon$ & $\epsilon$ & $\epsilon$ \\
\hline PLST & $\epsilon$ & $\epsilon$ & $\bar{\epsilon}$ & $\epsilon$ & $\bar{\epsilon}$ & $\epsilon$ & $\bar{\epsilon}$ & $\epsilon$ & $\bar{\epsilon}$ & $\bar{\epsilon}$ & $\bar{\epsilon}$ & $\epsilon$ & $\epsilon$ & $\bar{\epsilon}$ & $\bar{\epsilon}$ \\
\hline CPLST & $\bar{\epsilon}$ & $\bar{\epsilon}$ & $\bar{\epsilon}$ & $\bar{\epsilon}$ & $\bar{\epsilon}$ & $\bar{\epsilon}$ & $\bar{\epsilon}$ & $\epsilon$ & $\bar{\epsilon}$ & $\bar{\epsilon}$ & $\bar{\epsilon}$ & $\bar{\epsilon}$ & $\bar{\epsilon}$ & $\bar{\epsilon}$ & $\bar{\epsilon}$ \\
\hline MLC-BMaD & $\epsilon$ & $\epsilon$ & $\epsilon$ & $\epsilon$ & $\epsilon$ & $\epsilon$ & $\epsilon$ & $\epsilon$ & $\epsilon$ & $\epsilon$ & $\epsilon$ & $\epsilon$ & $\epsilon$ & $\epsilon$ & $\bar{\epsilon}$ \\
\hline ML-CSSP & $\bar{\epsilon}$ & $\bar{\epsilon}$ & $\bar{\epsilon}$ & $\bar{\epsilon}$ & $\bar{\epsilon}$ & $\bar{\epsilon}$ & $\bar{\epsilon}$ & $\bar{\epsilon}$ & $\epsilon$ & $\epsilon$ & $\epsilon$ & $\epsilon$ & $\epsilon$ & $\epsilon$ & $\epsilon$ \\
\hline Datasets & \multicolumn{5}{|c|}{ ESPGame } & \multicolumn{5}{|c|}{ bibtex } & \multicolumn{5}{|c|}{ bookmarks } \\
\hline$d_{z} / d_{y}$ & $5 \%$ & $10 \%$ & $15 \%$ & $20 \%$ & $25 \%$ & $10 \%$ & $20 \%$ & $30 \%$ & $40 \%$ & $50 \%$ & $10 \%$ & $20 \%$ & $30 \%$ & $40 \%$ & $50 \%$ \\
\hline CS & $\epsilon$ & $\epsilon$ & $\epsilon$ & $\epsilon$ & $\epsilon$ & $\epsilon$ & $\epsilon$ & $\epsilon$ & $\epsilon$ & $\epsilon$ & $\bar{\epsilon}$ & $\epsilon$ & $\epsilon$ & $\epsilon$ & $\epsilon$ \\
\hline PLST & $\epsilon$ & $\epsilon$ & $\bar{\epsilon}$ & $\bar{\epsilon}$ & $\epsilon$ & $\epsilon$ & $\bar{\epsilon}$ & $\bar{\epsilon}$ & $\bar{\epsilon}$ & $\bar{\epsilon}$ & $\bar{\epsilon}$ & $\bar{\epsilon}$ & $\bar{\epsilon}$ & $\bar{\epsilon}$ & $\bar{\epsilon}$ \\
\hline CPLST & $\epsilon$ & $\epsilon$ & $\bar{\epsilon}$ & $\epsilon$ & $\epsilon$ & $\epsilon$ & $\bar{\epsilon}$ & $\bar{\epsilon}$ & $\bar{\epsilon}$ & $\bar{\epsilon}$ & $\bar{\epsilon}$ & $\epsilon$ & $\epsilon$ & $\bar{\epsilon}$ & $\bar{\epsilon}$ \\
\hline MLC-BMaD & $\epsilon$ & $\epsilon$ & $\bar{\epsilon}$ & $\epsilon$ & $\epsilon$ & $\epsilon$ & $\bar{\epsilon}$ & $\bar{\epsilon}$ & $\bar{\epsilon}$ & $\bar{\epsilon}$ & $\bar{\epsilon}$ & $\epsilon$ & $\epsilon$ & $\epsilon$ & $\bar{\epsilon}$ \\
\hline ML-CSSP & $\epsilon$ & $\bar{\epsilon}$ & $\bar{\epsilon}$ & $\bar{\epsilon}$ & $\bar{\epsilon}$ & $\bar{\epsilon}$ & $\bar{\epsilon}$ & $\bar{\epsilon}$ & $\epsilon$ & $\bar{\epsilon}$ & $\bar{\epsilon}$ & $\bar{\epsilon}$ & $\epsilon$ & $\bar{\epsilon}$ & $\bar{\epsilon}$ \\
\hline
\end{tabular}

the proposed method against those of any compared baseline, and calculate the differences between pairwise samples for significance tests. The null hypothesis $H_{0}$ here is that the mean value of such differences equals zero, and the alternative hypothesis $H_{\alpha}$ is that the mean value of such differences does not equal zero and the mean value of the F1/accuracy values yielded by $\mathrm{E}^{2} \mathrm{FE}$ is larger than that yielded by the compared baseline. We set the significance level as a typical value 0.01 . Then if a $P$-value generated by the significance test satisfies that $P$-value $\leq 0.01$, the null hypothesis $H_{0}$ will be rejected and the alternative hypothesis $H_{\alpha}$ is considered to be statistically significant.

Here Table I and II report all the $P$-values of the significance tests between $\mathrm{E}^{2} \mathrm{FE}$ and each baseline for both label-based macroFl and example-based Accuracy on all datasets with varying $d_{z} / d_{y}$. For clarity, here a $P$-value less than $10^{-10}$ is denoted as a tiny value $\epsilon$. From the results of significance tests, we can find that: 1) for label-based macroF1, except the cases where $\mathrm{E}^{2} \mathrm{FE}$ is compared against CS with $d_{z} / d_{y} \in\{20 \%, 25 \%\}$ on ESPGame, the maximal $P$-value in all significance tests between $\mathrm{E}^{2} \mathrm{FE}$ and the compared baselines is around $1.5 \times 10^{-3}, 2$ ) for example-based Accuracy, all $P$-values in significance tests are less than $10^{-10}$. Therefore, considering that nearly all $P$-values in the significance tests are less than the significance level, i.e. 0.01, the performance improvements gained by $\mathrm{E}^{2} \mathrm{FE}$ over the compared baselines are statistically significant.

\section{B. Experimental Validation for the Orthonormality Assump-} tion in $E^{2} F E$

As mentioned in the paper (section VIII), to further demonstrate the reasonableness of introducing the orthonormality assumption into the proposed $\mathrm{E}^{2} \mathrm{FE}$, we also try dropping it from the objective function and conduct experiments to see how it affects the classification performance and the computational costs of $\mathrm{E}^{2} \mathrm{FE}$. We denote this case as $\mathrm{E}^{2} \mathrm{FE}_{\text {NoOrth }}$. Specifically, without the orthonormality assumption, the objective function of $\mathrm{E}^{2} \mathrm{FE}_{\text {NoOrth }}$ will be as follows.

$$
\begin{aligned}
& \Psi=\max _{\mathbf{Z}, \mathbf{Q}}-\|\mathbf{Y}-\mathbf{Z Q}\|_{\text {fro }}^{2}+\alpha \operatorname{Tr}\left[\mathbf{Z}^{T} \mathbf{H Z}\right] \\
& =\min _{\mathbf{Z}, \mathbf{Q}}\|\mathbf{Y}-\mathbf{Z} \mathbf{Q}\|_{\text {fro }}^{2}-\alpha \operatorname{Tr}\left[\mathbf{Z}^{T} \mathbf{H Z}\right] \\
& \text { s.t. } \quad \forall i \in\left\{1,2, \ldots, d_{z}\right\}, \quad \mathbf{Z}_{\cdot, i}^{T} \mathbf{Z}, i=1
\end{aligned}
$$

where $\mathbf{Y}$ is the tagging matrix of training instances, $\mathbf{Z}$ and $\mathbf{Q}$ are respectively the to-be-learnt code matrix and the linear decoding matrix, and $\mathbf{H}=\mathbf{X}\left(\mathbf{X}^{T} \mathbf{X}\right)^{-1} \mathbf{X}^{T}$ with $\mathbf{X}$ being the feature matrix of training instances. Note that $\mathbf{Z}_{\cdot, i}^{T} \mathbf{Z}_{\cdot, i}=1$ is still required here as it is the precondition for $\operatorname{Tr}\left[\mathbf{Z}^{T} \mathbf{H Z}\right]$ being an expression of predictability, as described in section III-B2. Like other matrix factorization methods [2], [3], we can derive the code matrix $\mathbf{Z}$ and the linear decoding matrix $\mathbf{Q}$ via iteratively and alternatively optimizing one with the other fixed, though that cannot ensure to obtain the global optima. Specifically, with $\mathbf{Z}$ fixed, $\mathbf{Q}$ can be optimized as $\mathbf{Q}=\left(\mathbf{Z}^{T} \mathbf{Z}\right)^{-1} \mathbf{Z}^{T} \mathbf{Y}$. Meanwhile, with $\mathbf{Q}$ fixed, the gradient w.r.t $\mathbf{Z}$ can be calculated as $\frac{\partial \Psi}{\partial \mathbf{Z}}=-2(\mathbf{Y}-\mathbf{Z Q}) \mathbf{Q}^{T}-2 \alpha \mathbf{H Z}$. Considering the constraints for each column of $\mathbf{Z}$, it is infea- 
TABLE III

Performance Comparisons BETWEen E ${ }^{2} \mathrm{FE}$ AND E $\mathrm{F}^{2} \mathrm{FE}_{\text {NoOrth }}$ ON ALL DATASETS, WITH $p=5$ FOR ESPGame AND $p=10$ FOR OTHERS.

\begin{tabular}{|c|c|c|c|c|c|c|c|}
\hline & & $d_{z} / d_{y}$ & $p \%$ & $2 p \%$ & $3 p \%$ & $4 p \%$ & $5 p \%$ \\
\hline \multirow{12}{*}{ 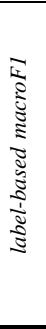 } & \multirow{2}{*}{ delicious } & $\mathrm{E}^{2} \mathrm{FE}$ & 0.0530 & 0.0569 & 0.0577 & 0.0578 & 0.0578 \\
\hline & & $\mathrm{E}^{2} \mathrm{FE}_{\text {NoOrth }}$ & 0.0336 & 0.0401 & 0.0444 & 0.0462 & 0.0438 \\
\hline & \multirow{2}{*}{ CAL500 } & $\mathrm{E}^{2} \mathrm{FE}$ & 0.1198 & 0.1247 & 0.1263 & 0.1258 & 0.1256 \\
\hline & & $\mathrm{E}^{2} \mathrm{FE}_{\text {NoOrth }}$ & 0.0966 & 0.1086 & 0.1074 & 0.1122 & 0.1120 \\
\hline & \multirow{2}{*}{ mediamill } & $\mathrm{E}^{2} \mathrm{FE}$ & 0.0549 & 0.0575 & 0.0577 & 0.0577 & $\mathbf{0 . 0 5 7 7}$ \\
\hline & & $\mathrm{E}^{2} \mathrm{FE}_{\text {NoOrth }}$ & 0.0436 & 0.0448 & 0.0459 & 0.0451 & 0.0453 \\
\hline & \multirow{2}{*}{ ESPGame } & $\mathrm{E}^{2} \mathrm{FE}$ & 0.0026 & 0.0025 & 0.0025 & 0.0025 & 0.0025 \\
\hline & & $\mathrm{E}^{2} \mathrm{FE}_{\text {NoOrth }}$ & 0.0018 & 0.0018 & 0.0017 & 0.0018 & 0.0018 \\
\hline & \multirow{2}{*}{ bibtex } & $\mathrm{E}^{2} \mathrm{FE}$ & 0.0595 & 0.0888 & 0.1169 & 0.1286 & 0.1369 \\
\hline & & $\mathrm{E}^{2} \mathrm{FE}_{\text {NoOrth }}$ & 0.0420 & 0.0727 & 0.0847 & 0.0929 & 0.1000 \\
\hline & \multirow{2}{*}{ bookmarks } & $\mathrm{E}^{2} \mathrm{FE}$ & 0.0472 & 0.0706 & 0.0752 & 0.0764 & 0.0775 \\
\hline & & $\mathrm{E}^{2} \mathrm{FE}_{\text {NoOrth }}$ & 0.0298 & 0.0455 & 0.0459 & 0.0471 & 0.0488 \\
\hline \multirow{12}{*}{ 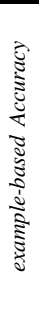 } & \multirow{2}{*}{ delicious } & $\mathrm{E}^{2} \mathrm{FE}$ & 0.1187 & 0.1196 & 0.1197 & 0.1196 & 0.1184 \\
\hline & & $\mathrm{E}^{2} \mathrm{FE}_{N o O r t h}$ & 0.1037 & 0.1073 & 0.1082 & 0.1093 & 0.1045 \\
\hline & \multirow{2}{*}{ CAL500 } & $\mathrm{E}^{2} \mathrm{FE}$ & 0.2405 & 0.2411 & 0.2421 & 0.2396 & 0.2392 \\
\hline & & $\mathrm{E}^{2} \mathrm{FE}_{\text {NoOrth }}$ & 0.2261 & 0.2294 & 0.2256 & 0.2267 & 0.2250 \\
\hline & \multirow{2}{*}{ mediamill } & $\mathrm{E}^{2} \mathrm{FE}$ & 0.4353 & 0.4379 & 0.4378 & 0.4378 & 0.4378 \\
\hline & & $\mathrm{E}^{2} \mathrm{FE}_{\text {NoOrth }}$ & 0.4192 & 0.4201 & 0.4204 & 0.4192 & 0.4199 \\
\hline & \multirow{2}{*}{ ESPGame } & $\mathrm{E}^{2} \mathrm{FE}$ & 0.0701 & 0.0701 & 0.0700 & 0.0701 & 0.0701 \\
\hline & & $\mathrm{E}^{2} \mathrm{FE}_{N o O r t h}$ & 0.0613 & 0.0635 & 0.0605 & 0.0585 & 0.0590 \\
\hline & \multirow{2}{*}{ bibtex } & $\mathrm{E}^{2} \mathrm{FE}$ & 0.1835 & 0.2149 & 0.2356 & 0.2440 & 0.2493 \\
\hline & & $\mathrm{E}^{2} \mathrm{FE}_{\text {NoOrth }}$ & 0.1501 & 0.2018 & 0.2121 & 0.2167 & 0.2233 \\
\hline & \multirow{2}{*}{ bookmarks } & $\mathrm{E}^{2} \mathrm{FE}$ & 0.1659 & 0.1913 & 0.1933 & 0.1937 & 0.1939 \\
\hline & & $\mathrm{E}^{2} \mathrm{FE}_{\text {NoOrth }}$ & 0.1521 & 0.1627 & 0.1636 & 0.1645 & 0.1660 \\
\hline
\end{tabular}

TABLE IV

AverAgE TIME COSTS (IN SECONDS) FOR PERFORMING LSDR IN E ${ }^{2} \mathrm{FE}$ AND E ${ }^{2} \mathrm{FE}_{N o O r t h}$ WITH $d_{z} / d_{y}=10 \%$.

\begin{tabular}{l|l|l|l} 
& delicious & ESPGame & bookmarks \\
\hline \hline $\mathrm{E}^{2} \mathrm{FE}$ & 1.984 & 10.877 & 4.877 \\
\hline $\mathrm{E}^{2} \mathrm{FE}_{\text {NoOrth }}$ & 145.253 & 215.199 & 41.434 \\
\hline
\end{tabular}

not bring substantial performance improvements and instead will increase the time costs for optimization. On the contrary, keeping the orthonormality assumption gains a good trade-off between efficiency and effectiveness for LSDR.

\section{REFERENCES}

[1] J. McDonald, Handbook of Biological Statistics (3rd ed.). Sparky House Publishing, 2014

[2] G. Ding, Y. Guo, and J. Zhou, "Collective matrix factorization hashing for multimodal data," in The IEEE Conference on Computer Vision and Pattern Recognition, 2014.

[3] X. He, H. Zhang, M.-Y. Kan, and T.-S. Chua, "Fast matrix factorization for online recommendation with implicit feedback," in Proceedings of the 39th International ACM SIGIR Conference on Research and Development in Information Retrieval, 2016. sible to derive a closed-form solution for the optimal $\mathbf{Z}$. And thus in our experiments we utilize a gradient descent based method for optimizing $\mathbf{Z}$, which always takes one appropriate step to make the objective function decrease while keeping the constraints satisfied. By iteratively optimizing $\mathbf{Z}$ and $\mathbf{Q}$ until convergence (i.e. the relative change of the objective function is less than $0.1 \%$ in our experiments), we utilize them to train predictive models and then perform classification for unseen instances.

The performance of $\mathrm{E}^{2} \mathrm{FE}_{\text {NoOrth }}$ on all datasets, using random initial values for $\mathbf{Z}$ and $\mathbf{Q}$ to perform optimization, is presented in Table III. It can be seen that $\mathrm{E}^{2} \mathrm{FE}_{\text {NoOrth }}$ is inferior to $E^{2} F E$. In fact, even using the derived $\mathbf{Z}$ and $\mathrm{Q}$ of $\mathrm{E}^{2} \mathrm{FE}$ as initial values, $\mathrm{E}^{2} \mathrm{FE}_{N o \text { Orth }}$ can hardly gain performance improvement over $\mathrm{E}^{2} \mathrm{FE}$. It is mainly attributed to that, without the orthonormality assumption, 1) more redundant information rather than complementary information exists between dimensions of the latent space, and 2) global optima cannot be ensured for $\mathbf{Z}$.

Moreover, we also evaluate the time costs for $\mathrm{E}^{2} \mathrm{FE}_{N o O r t h}$ to perform LSDR on the largest delicious, ESPGame and bookmarks with $d_{z} / d_{y}=10 \%$, as reported in Table IV. We can see that $\mathrm{E}^{2} \mathrm{FE}_{\text {NoOrth }}$ costs much more time than $\mathrm{E}^{2} \mathrm{FE}$, because its objective function is more complex for optimization. Its time costs are even higher than those of the binary relevance model L-RR (see Table VIII in the paper), which means that $\mathrm{E}^{2} \mathrm{FE}_{\text {NoOrth }}$ may be unsuitable for LSDR.

Therefore, dropping the orthonormality assumption does 